\title{
Self-Trapped Excitons in Ionic-Covalent Silver Halide Crystals and Nanostructures: High-Frequency EPR, ESE, ENDOR and ODMR Studies
}

\author{
P. G. Baranov • N. G. Romanov • O. G. Poluektov • \\ J. Schmidt
}

Received: 24 September 2010/Published online: 29 October 2010

(C) The Author(s) 2010. This article is published with open access at Springerlink.com

\begin{abstract}
Silver halides have unique features in solid state physics because their properties are considered to be of borderline nature between ionic and covalent bonding. In $\mathrm{AgCl}$, the self-trapped hole ( $\mathrm{STH})$ is centered and partly trapped in the cationic sublattice, forming an $\mathrm{Ag}^{2+}$ ion inside of a $\left(\mathrm{AgCl}_{6}\right)^{4-}$ complex as a result of the Jahn-Teller distortion. The STH in $\mathrm{AgCl}$ can capture an electron from the conduction band forming the self-trapped exciton (STE). Recent results of a study of STE by means of high-frequency electron paramagnetic resonance, electron spin echo, electron-nuclear double resonance (ENDOR) and optically detected magnetic resonance (ODMR) are reviewed. The properties of the STE in AgCl crystals, such as exchange coupling, the ordering of the triplet and singlet sublevels, the dynamical properties of the singlet and triplet states, and the hyperfine interaction with the $\mathrm{Ag}$ and $\mathrm{Cl}(\mathrm{Br})$ nuclei are discussed. Direct information about the spatial distribution of the wave function of STE unpaired electrons was obtained by ENDOR. From a comparison with the results of an ENDOR study of the shallow electron center and STH, it is concluded that the electron is mainly contained in a hydrogen-like $1 s$ orbital with a Bohr radius of $15.1 \pm 0.6 \AA$, but near its center the electron density reflects the charge distribution of the hole. The hole of the STE is virtually identical to an isolated STH center. For $\mathrm{AgCl}$ nanocrystals embedded into the $\mathrm{KCl}$ crystalline matrix, the anisotropy of the $g$-factor of STE and STH was found to be substantially reduced compared with that of bulk $\mathrm{AgCl}$ crystals, which can be
\end{abstract}

P. G. Baranov · N. G. Romanov $(\bowtie)$

Ioffe Physical-Technical Institute, Russian Academy of Sciences,

194021 St. Petersburg, Russia

e-mail: nikolai.romanov@mail.ioffe.ru

O. G. Poluektov

Argonne National Laboratory, 9700 South Cass Avenue, Argonne, IL 60439, USA

J. Schmidt

Huygens Laboratory, Leiden University, 2300 RA Leiden, The Netherlands 
explained by a considerable suppression of the Jahn-Teller effect in nanoparticles. A study of ODMR in $\mathrm{AgBr}$ nanocrystals in $\mathrm{KBr}$ revealed spatial confinement effects and allowed estimating the nanocrystal size from the shape of the ODMR spectra.

\section{Introduction}

Self-trapping of charge carriers in the deformable lattice of a solid was predicted by Landau in 1933 for electrons [1] but among the best-known examples are the selftrapped holes (STHs) found in alkali halides and later in silver halides [2-9]. Silver halides have some unique features in solid state physics because their properties are considered to be of borderline nature between ionic and covalent bonding, that is, they are located close to the boundary corresponding to the critical value of ionicity 0.785, namely, 0.856 and 0.850 for $\mathrm{AgCl}$ and $\mathrm{AgBr}$, respectively [10]. In contrast to the alkali halides where the $\mathrm{STH}$ ( $V_{\mathrm{k}}$ center) is formed in the anionic sublattice and resides on two halide ions, in $\mathrm{AgCl}$ the hole is centered and partly trapped in the cationic sublattice, forming an $\mathrm{Ag}^{2+}$ ion inside of a $\left(\mathrm{AgCl}_{6}\right)^{4-}$ complex. The ground state of the $\mathrm{Ag}^{2+}\left(4 d^{9}\right)$ ion is a ${ }^{2} \mathrm{D}$ configuration and the corresponding energy level has fivefold degeneracy. In a cubic crystal field the level is split into a twofold $\left(\mathrm{E}_{\mathrm{g}}\right)$ and a threefold $\left(\mathrm{T}_{2 \mathrm{~g}}\right)$ degenerate level. These levels are split further due to a static Jahn-Teller distortion, which lowers the local symmetry to $D_{4 h}$ by elongation of the complex along a cubic axis.

Under ultraviolet (UV) light irradiation of $\mathrm{AgCl}$ and $\mathrm{AgBr}$ an electron is excited from the valence band into the conduction band and a hole is left in the valence band. In $\mathrm{AgCl}$, a hole is self-trapped on an $\mathrm{Ag}^{+}$ion in a $d\left(x^{2}-y^{2}\right)$-type orbital as the result of a Jahn-Teller distortion of the Ag-ion coordination sphere along a cubic axis. The free electron can be captured by a Coulombic core to form shallow electron centers (SEC), which are believed to play an important role in the latent image formation process, or by STH to form self-trapped exciton (STE).

Although the STH is centered and partly located at an $\mathrm{Ag}^{+}$ion, its wave function also contains contributions from the $3 s$ and $3 p$ orbitals of the neighboring $\mathrm{Cl}^{-}$ions in the plane perpendicular to the distortion axis. In forming the STE, the hole is expected to weakly bind an electron, because of the high dielectric constant of $\mathrm{AgCl}$. Recombination of the STE contributes to the broad luminescence band of $\mathrm{AgCl}$ crystals at low temperatures, which peaks at about $500 \mathrm{~nm}$, as demonstrated by studies using the method of optically detected magnetic resonance (ODMR). The properties of the excited states of this STE have been the subject of many ODMR investigations [11-19]. However, a number of important questions remained unclear: (1) the structure of the energy levels for STE; (2) the exchange interaction between an electron and a hole in the STE, the ordering of the singlet and triplet sublevels; (3) the dynamical properties of the excited singlet and triplet states; (4) the hyperfine (HF) interaction with the $\mathrm{Ag}$ and $\mathrm{Cl}$ nuclei; (5) the space distribution of the unpaired electron wave function; (6) the proofs of intrinsic nature of STH and STE; (7) the structure of STE as an STH coupled with an SEC. These problems cannot be solved with ODMR techniques at conventional microwave frequencies, because many resonance lines overlap (or partly overlap), and further because it is 
difficult to extract the dynamical properties from the continuous-wave ODMR spectra.

The STH and STE in $\mathrm{AgCl}$ are classical Jahn-Teller systems. The Jahn-Teller effect (JTE) is one of the fundamental local effects in the solid state, which is quite sensitive to internal fields and variations in electron-phonon interactions. Because of this, experimental and theoretical studies of the problem of the influence of nanoparticle size on the JTE are of great interest.

In this review the results of recent studies of bulk silver halides by means of ODMR, time-resolved electron paramagnetic resonance (EPR), electron spin echo (ESE), and electron-nuclear double resonance (ENDOR) at high-frequency $95 \mathrm{GHz}$ are presented. Owing to the high-energy resolution that is achieved at such high frequencies and the time-resolved character of the experiment, it was possible to obtain detailed new information about the STE, STH and SEC in the silver chloride crystals. Application of ODMR made it possible to study silver halide nanocrystals embedded in an ionic crystal matrix.

\section{Self-Trapped Excitons}

\subsection{The Energy Levels of Self-Trapped Excitons in $\mathrm{AgCl}$}

The existence of a singlet state of STE in AgCl separated by $1-25 \mathrm{~cm}^{-1}$ from the triplet state was assumed in Ref. [15]. In Ref. [16], the singlet state and the negative sign of the fine structure parameter $D$ of the triplet state were suggested from the analysis of the dependence of ODMR intensity on the microwave chopping frequency. The singlet-to-triplet splitting was estimated to be $6-7 \mathrm{~cm}^{-1}$ in Ref. [16]. Up to 1992 only the triplet STE state was studied by ODMR. Two different experiments allowed to confirm the existence of the singlet state and to measure very precisely the triplet-to-singlet exchange splitting, namely, W-band $(95 \mathrm{GHz})$ ODMR [20, 21] and multiquantum Q-band (35 GHz) ODMR [22, 23$].$

In Fig. 1a the optically detected EPR spectrum is shown, which was observed at $95 \mathrm{GHz}$ in the total luminescence of the $\mathrm{AgCl}$ crystal upon UV irradiation, with the magnetic field $B$ parallel to one of the cubic axes $\langle 100\rangle$ of the $\mathrm{AgCl}$ crystal. The transitions at 3.1572 and $3.3250 \mathrm{~T}$ correspond to resonances of the STH, with $B_{0}$ parallel to the distortion $z$-axis and $B \| x, y: g$ values $g_{\| \mathrm{STH}}=2.147$ and $g_{\perp \mathrm{STH}}=2.040$, respectively, in agreement with previous studies [7-9]. The two lines at 3.3541 and $3.3865 \mathrm{~T}$ correspond to the low-field and high-field transitions of the STE, with $B_{0}$ parallel to the distortion $z$-axis: they are characterized by $g_{\| \mathrm{STE}}=2.014$. The two lines at 3.4460 and $3.4782 \mathrm{~T}$ are the low-field and highfield transitions of the other two sites of the STE with $B \| x, y$ : they are characterized by a $g$ value $g_{\perp \mathrm{STE}}=1.960$. The resonance of the SEC at 3.6061 $\mathrm{T}$ has an isotropic $g$ value $g_{\text {SEC }}=1.881$.

The ODMR spectra of $\mathrm{AgCl}$ demonstrate the advantage of working at high microwave frequencies. The resonance lines of the STH, the STE, and the SEC in $\mathrm{AgCl}$ are separated so well that it is easy to follow their dependence on the orientation of $B$ with respect to the crystal axes. 
Fig. 1 a ODMR spectrum recorded at $95 \mathrm{GHz}$ in the total luminescence of the $\mathrm{AgCl}$ crystal upon UV irradiation, with the magnetic field $B$ parallel to one of the cubic axes $\langle 100\rangle$. The microwave was amplitude modulated at $f_{\mathrm{m}}=70 \mathrm{~Hz} . T=1.2 \mathrm{~K}$. b $95 \mathrm{GHz}$ ODMR obtained by sweeping the magnetic field over a broad range. The energy level scheme for STE and the observed transitions are shown in the upper part of the figure. c Energy level scheme for STE in $\mathrm{AgCl}$ with some of the observed multiquantum singletto-triplet transitions ( $n$ denotes the number of microwave quanta) and the ODMR signals measured at a high microwave power $(900 \mathrm{~mW})$ and at two different microwave frequencies: $35.23 \mathrm{GHz}$ (black arrows on the energy scheme) and $35.65 \mathrm{GHz}$ (open arrows). $f_{\mathrm{m}}=80 \mathrm{~Hz}, T=1.8 \mathrm{~K}$, $B \|[100]$ (a)
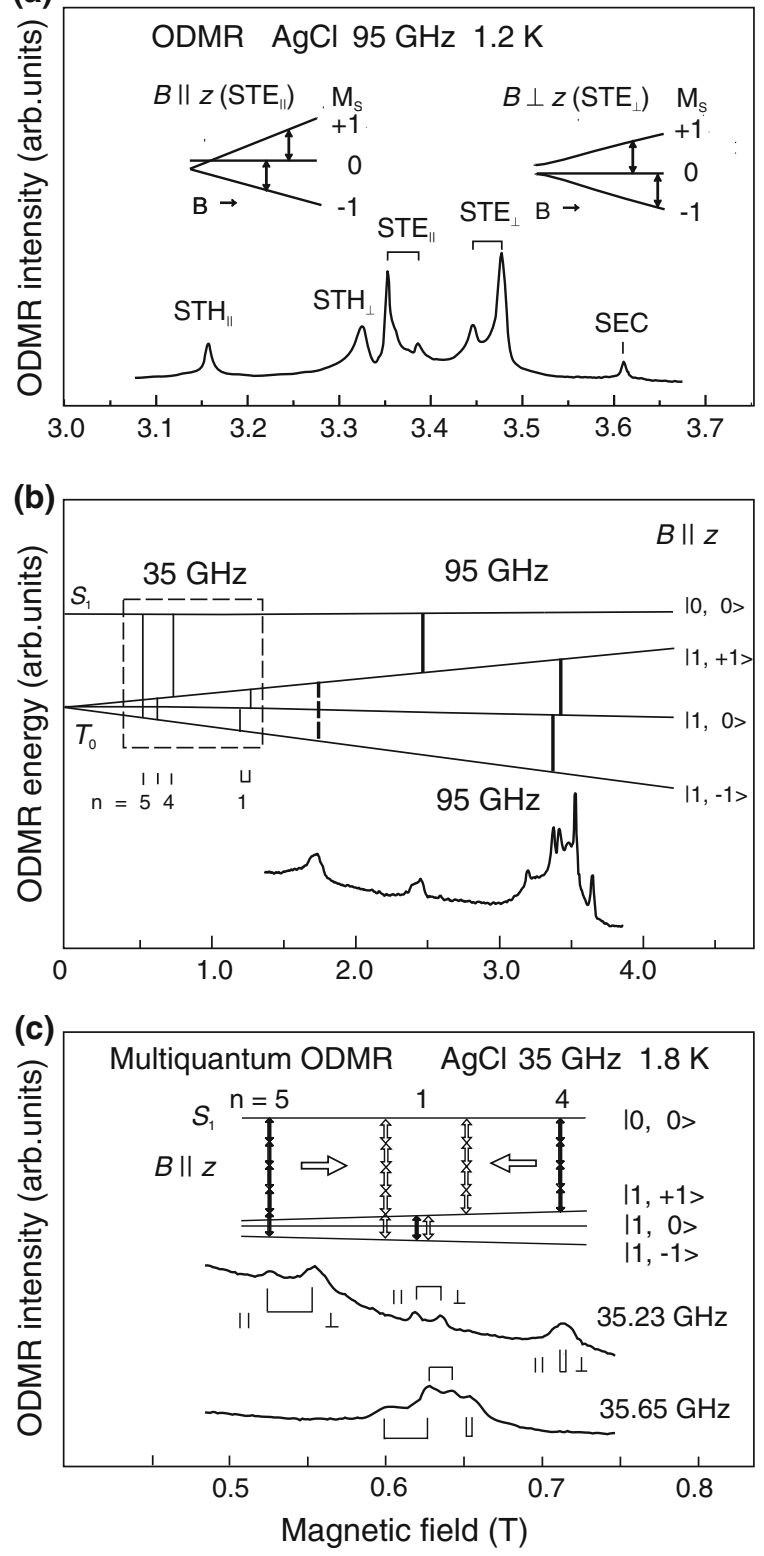

It was shown in Ref. [20] that the positions and the angular dependence of $95 \mathrm{GHz}$ ODMR lines of the STE considerably deviate from those calculated with a simplified $S=1$ spin Hamiltonian, which was used to treat the ODMR data at conventional X-and Q-band frequencies. At the high magnetic field of 3.3-3.5 T, at which the experiments at W-band have been performed, the triplet state of the STE cannot be considered as isolated from its accompanying singlet state. As a result, the 
$M_{S}=0$ sublevel of the triplet state is mixed with the singlet state. This leads, first of all, to a shift of the EPR transitions of the triplet state, which can be used for a determination of the value of the singlet-triplet splitting.

To explain the observed pattern of the resonance lines of the STE a more general spin Hamiltonian should be considered

$$
\hat{H}=\beta_{\mathrm{e}} \vec{B} \cdot \overleftrightarrow{\mathrm{g}}_{\mathrm{e}} \cdot \vec{s}_{\mathrm{e}}+\beta_{\mathrm{e}} \vec{B} \cdot \overleftrightarrow{g}_{\mathrm{h}} \cdot \vec{s}_{\mathrm{h}}+\vec{s}_{\mathrm{e}} \cdot \stackrel{\leftrightarrow}{D} \cdot \vec{s}_{\mathrm{h}}+J \vec{s}_{\mathrm{e}} \cdot \vec{s}_{\mathrm{h}}
$$

Here, the first two terms represent the Zeeman interaction of the electron and the hole forming STE. The third term describes the electron-hole spin-spin interaction and the last term describes their exchange interaction. The principal values of the $g$-tensor for the hole are $g_{\mathrm{h} z}=g_{\mathrm{h} \|}$ and $g_{\mathrm{h} x}=g_{\mathrm{h} y}=g_{\mathrm{h} \perp}$, the $g$-factor of the electron $g_{\mathrm{e}}$ is isotropic. Hyperfine interactions are not included in Eq. (1).

In the first high-frequency ODMR experiments the exchange splitting $J$ was derived, indirectly, via an analysis of the resonance fields of the ODMR transitions by taking into account the magnetic-field-induced mixing between the singlet state $\mathrm{S}_{1}(S=0)$ and the triplet state $\mathrm{T}_{0}\left(M_{S}=0\right)$ sublevel of the triplet state $S=1$ [20]. Such a mixing makes the transition between the $M_{S}=1$ triplet sublevel and $S_{1}$ slightly allowed. Indeed, a careful search revealed a resonance line in the ODMR spectrum which was attributed to this transition, as can be seen in Fig. 1b. Figure 1b shows the ODMR spectrum observed in the total luminescence of the $\mathrm{AgCl}$ crystal upon UV irradiation. The microwave power at $95 \mathrm{GHz}$ was amplitude modulated at $70 \mathrm{~Hz}$ for lock-in detection and the magnetic field was directed parallel to the crystal [100] axis. Figure $1 \mathrm{~b}$ clearly shows an ODMR line at the magnetic field of $2.41 \mathrm{~T}$. This line corresponds to the "forbidden" transition between the singlet state and the $M_{S}=+1$ sublevel of the triplet state of the STE. From its position the exchange coupling $J=-5.37 \pm 0.01 \mathrm{~cm}^{-1}$ was derived [21]. A line at $1.7 \mathrm{~T}$ is assigned to the $\Delta M_{S}=2$ transition.

Numerous multiquantum transitions corresponding to the absorption of up to seven microwave quanta (total energy $7 \times 35=245 \mathrm{GHz}$ ) were found in the $35 \mathrm{GHz}$ ODMR spectra of $\mathrm{AgCl}$ recorder in a wide magnetic field range from 0.5 to $4 \mathrm{~T}$ at a high $(>500 \mathrm{~mW})$ microwave power [22]. Two multiquantum singletto-triplet transitions corresponding to the absorption of 4 and 5 microwave quanta are marked in Fig. 1b. The assignment of the observed ODMR lines to the multiquantum transitions of the STE was unambiguously proved by the measurements of ODMR at slightly different microwave frequencies, i.e., with the different energy of the microwave quanta. Figure 1c shows a part of the ODMR spectrum recorded in $\mathrm{AgCl}$ at the microwave frequencies of 35.23 and $35.63 \mathrm{GHz}$, the microwave power of $900 \mathrm{~mW}$ and $T=1.8 \mathrm{~K}, B \|[100]$. The energy levels for $B \| z$ and the calculated positions of the EPR transitions for these two frequencies are shown in the upper part of the figure. One can see that the directions of the line shifts are different for different transitions and the value of the line shift is proportional to $n$ in complete agreement with calculations. Observations of the singlet-to-triplet multiquantum transitions for STE in $\mathrm{AgCl}$ allowed us to measure $J$ with extremely high accuracy: $J=5.370 \pm 0.002 \mathrm{~cm}^{-1}[22,23]$.

Although multiquantum ODMR has been observed by different groups in several systems (see Ref. [22] and references therein), a complete understanding of this effect is still missing. One of possible explanations takes into account multiquantum 
transitions via real intermediate electronic states which may appear as a result of vibronic interaction in a quasi-degenerate system of electronic states of the center. According to this mechanism both the electric $(E)$ and magnetic $(H)$ components of the microwave field can be active in the multiquantum transitions including an interference effect of $E$ and $H$.

The sign of the zero-field splitting parameter $D$ of the STE in $\mathrm{AgCl}$ was shown to be negative. This result confirms that the zero-field splitting in the triplet state of the STE is dominated by contributions of second-order spin-orbit coupling and that spin-spin (dipole-dipole) interactions are negligible.

\subsection{The Dynamical Properties of the Singlet and Triplet States of the STE}

The dynamical properties of the excited singlet and triplet states of the STE were studied in Ref. [21]. For this purpose, ESE technique in combination with pulsed laser excitation was used. The populating, depopulating, and spin-lattice relaxation processes have been studied. In addition, the results of ODMR experiments in magnetic field as well as in zero field which support the structure of STE as the STH-captured shallow electron were presented.

The ESE-detected EPR spectrum of the $\mathrm{AgCl}$ crystal at $94.9 \mathrm{GHz}$ with $B$ ॥ [001] is presented in Fig. 2. The crystal is first excited by a laser flash of the $\mathrm{XeCl}$ excimer laser at $308 \mathrm{~nm}$ (duration $10 \mathrm{ns)}$ ) and then subjected to a $(\pi / 2)-\tau-\pi$ microwave pulse sequence starting at a delay time $\tau_{\mathrm{d}}$ after the laser flash. The $\pi / 2$ - and $\pi$-pulse lengths are 30 and $60 \mathrm{~ns}$, respectively, $\tau=4 \mu \mathrm{s}$, and $\tau_{\mathrm{d}}=5 \mathrm{~ms}$. The spectrum is obtained by monitoring the echo height at a fixed value of $\tau_{\mathrm{d}}$ and $\tau$ and by sweeping the magnetic field. Two signals of the STH, four of the STE, and one of the SEC which are similar to those in the ODMR spectrum (Fig. 1) are recognized in the spectrum. We will concentrate on the signals of the STE.

First, the dephasing time $T_{2}$ of the signals in the spectrum displayed in Fig. 2 was measured. The results are listed in Table 1 . The remarkable finding is that the value of $T_{2}$ for the STE is about five times shorter than that for the STH and the SEC. In addition, the spin-lattice relaxation time $T_{1}$ of all ground-state paramagnetic centers was estimated via the variation of the signal intensity with the repetition rate. These values are also given in Table 1.

Second, the evolution of the spectrum of the STE by varying the delay time $\tau_{\mathrm{d}}$ after the laser flash was recorded for the delay times varying from 0.003 to $80 \mathrm{~ms}$ (see Fig. 2b). The striking aspect is that at the shorter delay time the signals, which are proportional to the population differences of the triplet sublevels connected by the microwave pulses, are zero. This means that within the experimental accuracy the populating rates of the sublevels are equal. The evolution of the signals is determined by the combined effect of decay and relaxation processes.

As mentioned already, the laser flash populates the sublevels $M_{S}=+1,0$ and -1 equally. Then in the first $0.5 \mathrm{~ms}$, the high-field and low-field signals of the $\mathrm{STE}_{\|}$ and of the $\mathrm{STE}_{\perp}$ start to develop equal absorptive intensities at the similar rates. This effect can only be understood by assuming that a dominant relaxation is present which transfers population from the $M_{S}=1$ to the $M_{S}=-1$ sublevels. It is interesting to note that this rate is temperature independent, which is in agreement 

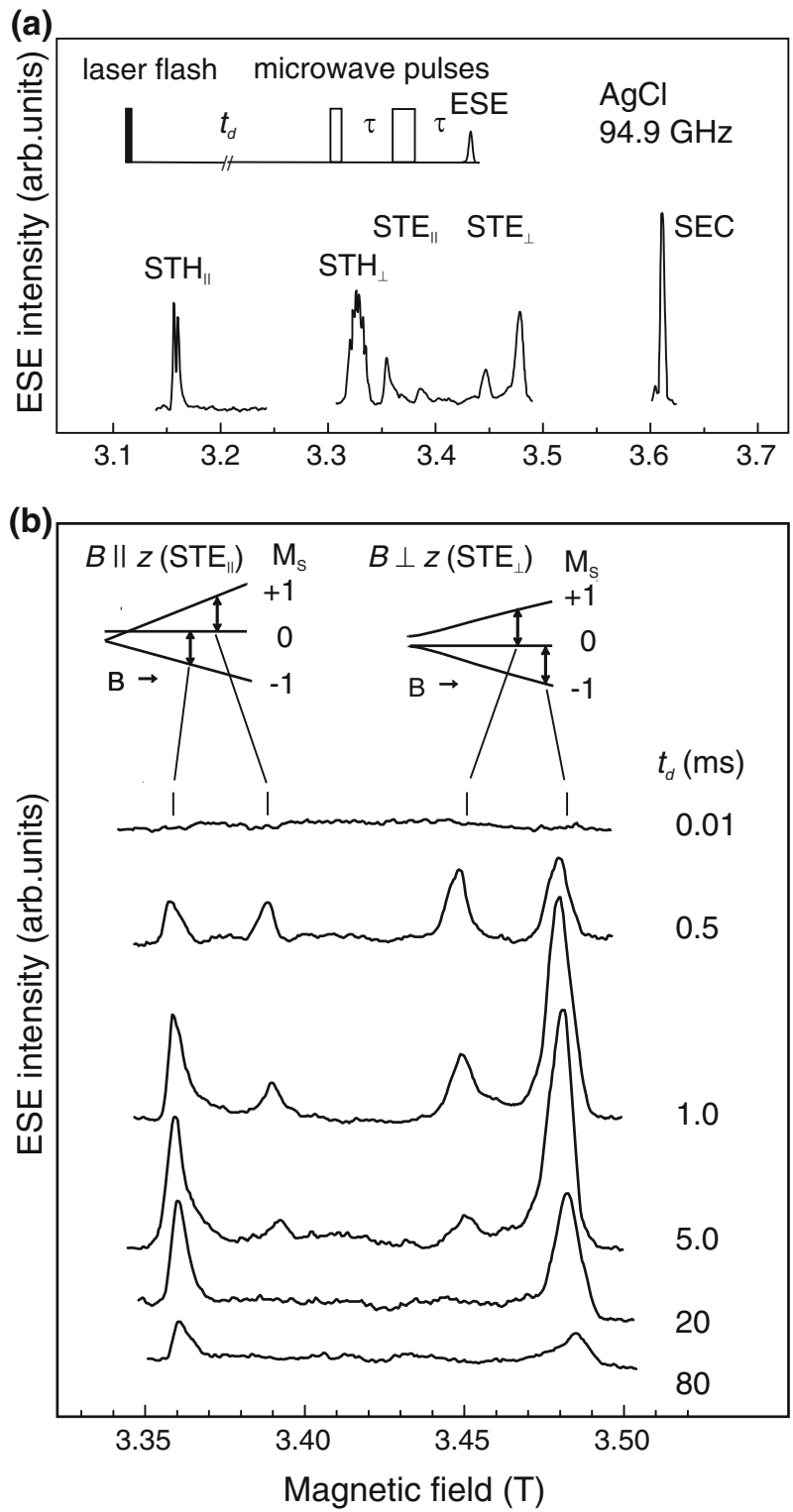

Fig. 2 a ESE-detected EPR spectrum of the $\mathrm{AgCl}$ crystal at $94.9 \mathrm{GHz}$ with $B \|$ [001] upon excitation by a laser flash at $308 \mathrm{~nm}$ (duration $10 \mathrm{~ns}$ ). The $(\pi / 2)-\tau-\pi$ microwave pulse sequence starts at a time $\tau_{\mathrm{d}}$ after the laser flash. The $\pi / 2$ - and $\pi$-pulse lengths are 30 and $60 \mathrm{~ns}$, respectively, $\tau=4 \mu \mathrm{s}$, and $\tau_{\mathrm{d}}=5 \mathrm{~ms}, T=1.2 \mathrm{~K}$. b The ESE-detected EPR spectrum of the STE as a function of the delay $\tau_{\mathrm{d}}$ after the laser flash

with the idea that this transfer corresponds to a direct process in which a phonon of energy $\sim 190 \mathrm{GHz}$ is created.

In the time interval between 1 and $5 \mathrm{~ms}$ the low-field signal of the STE $\mathrm{I}_{\|}$and highfield signal of the $\mathrm{STE}_{\perp}$ is further increasing, but the high-field signal of the $\mathrm{STE}_{\|}$ 
Table 1 Spin-spin relaxation time $T_{2}$ and spin-lattice relaxation time $T_{1}$ of the $\mathrm{STH}$, $\mathrm{STE}$ and SEC in the $\mathrm{AgCl}$ crystal at $T=1.2 \mathrm{~K}$

\begin{tabular}{lcc}
\hline Center & $T_{2}(\mu \mathrm{s})$ & $T_{1}(\mathrm{~ms})$ \\
\hline $\mathrm{STH}_{\|}$ & $110 \pm 5$ & $10 \pm 2$ \\
$\mathrm{STH}_{\perp}$ & $110 \pm 5$ & $10 \pm 2$ \\
$\mathrm{STE}_{\|}$ & $17.5 \pm 1.5$ & $16.0 \pm 0.4(2 \mathrm{~K})$ \\
$\mathrm{STE}_{\perp}$ & $23.0 \pm 0.8$ & $20.4 \pm 0.5(2 \mathrm{~K})$ \\
$\mathrm{SEC}$ & $90 \pm 5$ & $10 \pm 2$ \\
\hline
\end{tabular}

and the low-field signal of the $\mathrm{STE}_{\perp}$ decreases to zero at the same rate. In the time interval between 5 and $100 \mathrm{~ms}$, only the low-field $\mathrm{STE}_{\|}$and high-field $\mathrm{STE}_{\perp}$ signals remain and start to decay at different rates. These rates become temperature independent below $1.3 \mathrm{~K}$.

The time-resolved ESE and ODMR experiments have allowed unraveling the populating and decaying processes of the triplet spin sublevels. The relative populating rates in zero field as well as in magnetic field were found to be equal. Apparently, upon excitation over the band gap, pairs of electrons and holes are formed and the probabilities of generating the singlet state or the three sublevels of the triplet state of the STE are equal. The remarkable observation was the presence of the dominant and temperature-independent relaxation rate from the $M_{S}=1$ to the $M_{S}=-1$ magnetic sublevels. It was proposed to be caused by a tunneling process in which the elongation of the STE changes direction from one cubic axis to another one. The consequence of this tunneling is that the expression for the zero-field Hamiltonian becomes time dependent and varies between $D S_{z}^{2}-1 / 3 S(S+1)$ and $D S_{x}^{2}-1 / 3 S(S+1)$ or $D S_{y}^{2}-1 / 3 S(S+1)$. The two latter forms contain the bilinear operators $S_{x}^{2}$ and $S_{y}^{2}$, which are capable of inducing selectively transitions between the $M_{S}=1$ and the $M_{S}=-1$ sublevels.

The value of $T_{2}$ shortens between 1.2 and $2.0 \mathrm{~K}$. This indicates that the tunneling process is not a purely coherent process but that a thermally activated contribution is present. Further, we note that $T_{2}$ for the parallel orientation is somewhat shorter than for the perpendicular one. This difference can be understood by the fact that in the parallel ( $z$ ) orientation, energy jumps (to $x$ or $y$ ) lead to a change in the resonance frequency and thus to a dephasing of the triplet spins. In contrast in the perpendicular orientation, only half of the jumps (to $z$ ) destroy the phase coherence.

\section{3 $\mathrm{HF}$ Interactions for the $\mathrm{STE}$ in $\mathrm{AgCl}$}

EPR and ODMR of the STE do not provide information on HF interactions since no resolved HF structure is observed. We, therefore, performed ENDOR spectroscopy at $94.9 \mathrm{GHz}$ using a method which is based on the ESE-detected ENDOR, and in addition, the ODMR measurements in the zero magnetic field.

Figure 3 shows the zero-field ODMR spectrum of the STE obtained at $T=1.2 \mathrm{~K}$ by scanning amplitude-modulated microwaves through resonance while detecting synchronously in the optical emission. The spectrum allows the observation of two components of the $\mathrm{T}_{0 z}-\mathrm{T}_{0 x, y}$ transitions between the triplet sublevels in zero magnetic field with a separation of $45 \pm 4 \mathrm{MHz}$. We attribute the dominant 


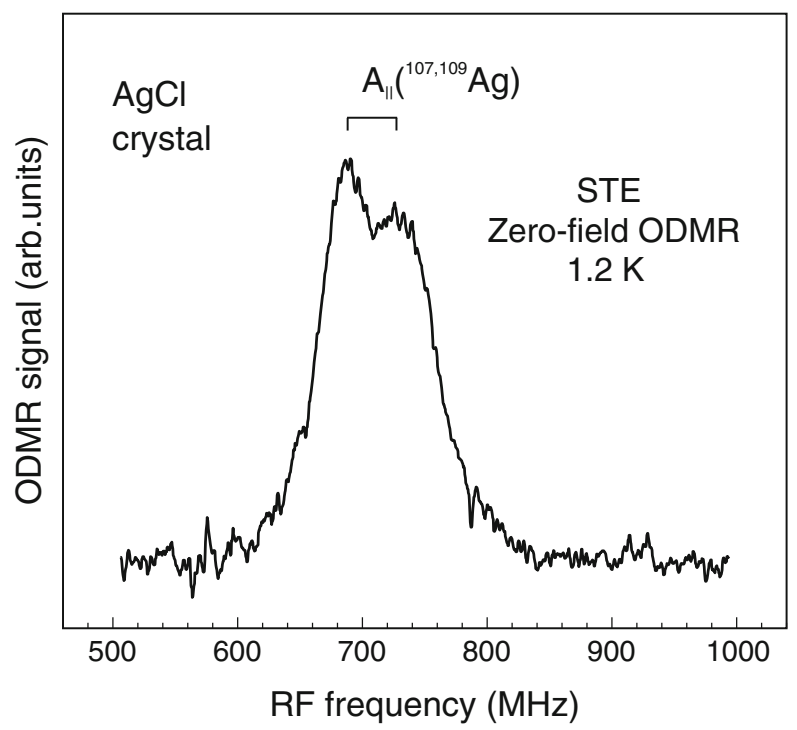

Fig. 3 Zero-field ODMR spectrum of the STE recorded by scanning the frequency of amplitudemodulated microwave field and lock-in detection of the emitted light. $f_{\mathrm{m}}=43 \mathrm{~Hz}, T=1.2 \mathrm{~K}$

contribution to this splitting to the HF-interaction term $A_{z z} S_{z} I_{z}$ of the triplet spin with the ${ }^{107} \mathrm{Ag}$ and ${ }^{109} \mathrm{Ag}$ nuclear spins. This term $A_{z z} S_{z} I_{z}$ gives matrix elements between $\mathrm{T}_{0 x}$ and $\mathrm{T}_{0 y}$ but since these two levels are degenerate it will lead to a first-order splitting of these levels equal to $A_{z z}$. The terms $A_{x x} S_{x} I_{x}$ and $A_{y y} S_{y} I_{y}$ only give secondorder shifts of the order of $2 \mathrm{MHz}$. Further, for the Cl nuclei, the term $A_{z z} S_{z} I_{z}$ is zero and the terms $A_{x x} S_{x} I_{x}$ and $A_{y y} S_{y} I_{y}$ also can give only second-order shifts of about $2 \mathrm{MHz}$. It is important to note that for the $\mathrm{STH}$, a hyperfine interaction with the $\mathrm{Ag}$ nuclear spins $A_{z z}=93 \pm 6 \mathrm{MHz}$ has been observed, i.e., almost exactly twice the value found for the STE. As a result it was concluded that this HF interaction in the STE is dominated by the electron spin of the STH and that the contribution of the spin of the bound electron is small since the electron in the STE is very delocalized and the density of its wave function at the position of the Ag nucleus is too small to be detected. Thus, the STH is proved to reflect the hole part of the STE.

Figure 4a shows the ESE-detected EPR spectrum of STE and SEC in the undoped $\mathrm{AgCl}$ crystal recorded with the magnetic field oriented along one of the cubic axes $(B \|[001])$ at $94.9 \mathrm{GHz}$ and $1.2 \mathrm{~K}$, under continuous UV irradiation. The spectrum is obtained by monitoring the height of the ESE signal detected at time $\tau$ after a $(\pi / 2)-\tau-\pi$ microwave pulse sequence. The $\pi / 2$ pulse length is $100 \mathrm{~ns}$, $\tau=650 \mathrm{~ns}$, and the repetition rate of the pulse sequence is $33 \mathrm{~Hz}$. The energy level diagrams for the two orientations of STE in magnetic field with $B$ parallel $\left(\mathrm{STE}_{\|}\right)$ and perpendicular $\left(\mathrm{STE}_{\perp}\right)$ to the distortion $z$-axis of the STE and for the SEC are presented on the top.

In Fig. 4b, two ENDOR spectra of the STE triplet state in $\mathrm{AgCl}$ are presented. For comparison the silver ENDOR spectrum of the shallow electron center is shown in Fig. 4c. The spectra were recorded at $94.9 \mathrm{GHz}$ and $1.2 \mathrm{~K}$ with $B$ along the cubic 

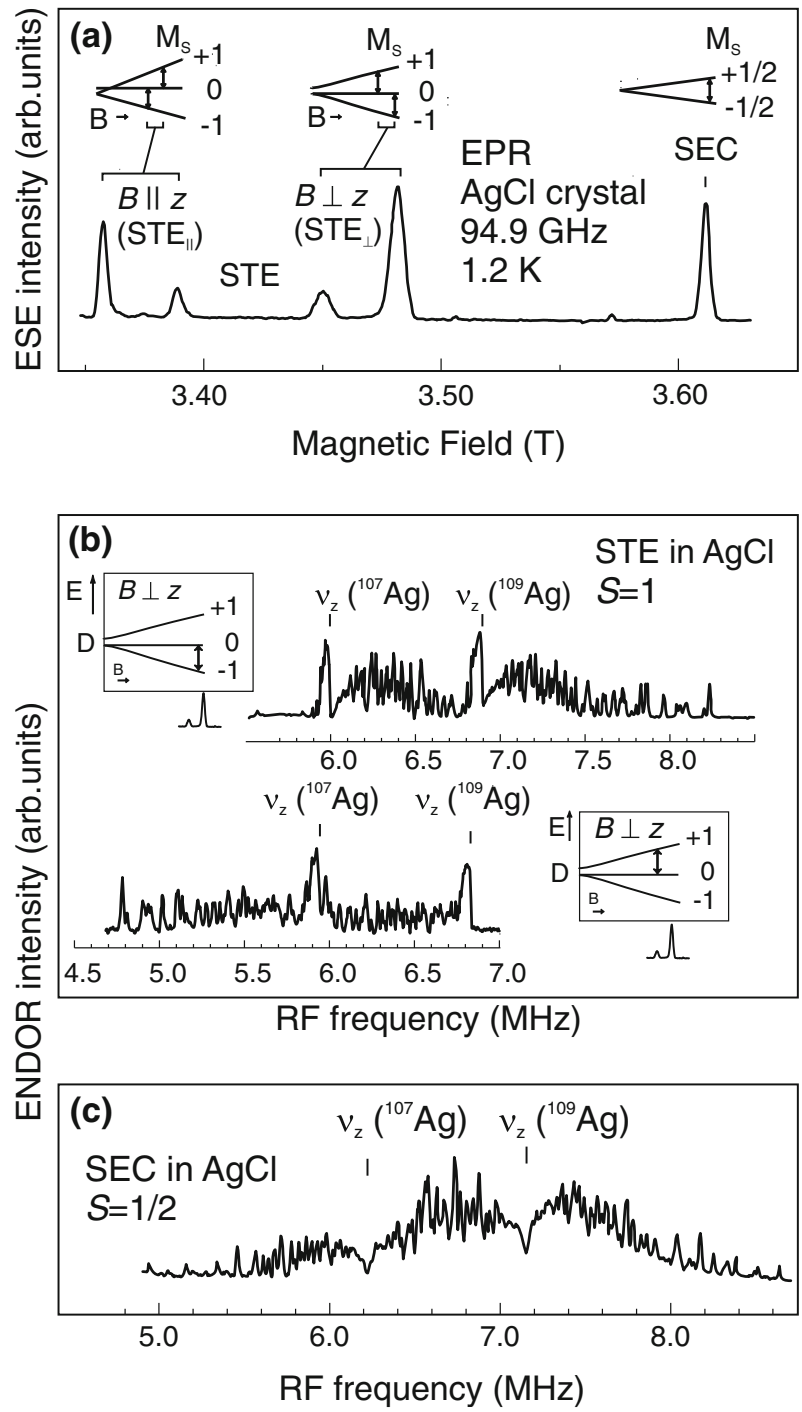

Fig. 4 a ESE-detected EPR spectrum of STE and SEC in undoped AgCl crystal recorded with $B \|$ [001] at $94.9 \mathrm{GHz}$ and $1.2 \mathrm{~K}$, during continuous UV irradiation. The spectrum is obtained by monitoring the height of the ESE signal created at time $\tau$ after a $(\pi / 2)-\tau-\pi$ microwave pulse sequence. The $\pi / 2$ pulse length is $100 \mathrm{~ns}, \tau=650 \mathrm{~ns}$, and the repetition rate of the pulse sequence is $33 \mathrm{~Hz}$. The energy levels diagrams for the two orientations of the STE in magnetic field with $B$ parallel $\left(\mathrm{STE}_{\|}\right)$and perpendicular $\left(\mathrm{STE}_{\perp}\right)$ to the distortion $z$-axis and for SEC are presented in the top. $\mathbf{b}$ Ag ENDOR spectra of the triplet state of the STE. The spectra were recorded at $94.9 \mathrm{GHz}$ and $1.2 \mathrm{~K}$ with $B$ along the cubic axis, which is perpendicular to the distortion axis of the $\mathrm{STE}\left(\mathrm{STE}_{\perp}\right)$ : the upper spectrum was obtained by monitoring the high-field EPR transition of $\mathrm{STE}_{\perp}$, which corresponds to the transition between the $M_{S}=0$ and $M_{S}=-1$ sublevels of the triplet and the lower spectrum was recorded via the low-field transition of $\mathrm{STE}_{\perp}$, which corresponds to the transition between $M_{S}=0$ and $M_{S}=+1$. c Silver ENDOR spectrum of the shallow electron center at $94.9 \mathrm{GHz}$ and $1.2 \mathrm{~K}$. Typical pulse lengths: $\pi / 2=100 \mathrm{~ns}, \tau$ between 400 and $900 \mathrm{~ns}, T=700 \mu$ s and the length of radio-frequency $(\mathrm{RF})$ pulse $=600 \mu \mathrm{s}$ 
axis, perpendicular to the distortion axis of the $\mathrm{STE}\left(\mathrm{STE}_{\perp}\right)$ : the upper spectrum in Fig. $4 \mathrm{~b}$ was obtained by monitoring the high-field EPR transition of $\mathrm{STE}_{\perp}$, which corresponds to the transition between the $M_{S}=0$ and $M_{S}=-1$ sublevels of the triplet and the lower spectrum was recorded via the low-field transition of $\mathrm{STE}_{\perp}$, which corresponds to the $M_{S}=0 \leftrightarrow M_{S}=+1$ transition. Here a $(\pi / 2)-\tau-\pi / 2-T-\pi / 2$ microwave pulse sequence is applied resonant with the part of the EPR signal of STE presented in the insets. Two groups of lines can be distinguished which both contain nuclear transitions of ${ }^{107} \mathrm{Ag}$ and ${ }^{109} \mathrm{Ag}$ nuclei. The first group covers a range of a few $\mathrm{MHz}$ and is positioned in Fig. $4 \mathrm{~b}$ above the nuclear Zeeman frequencies of ${ }^{107} \mathrm{Ag}$ and ${ }^{109} \mathrm{Ag}$ for the upper spectrum and below these frequencies for the lower spectrum. The second group covers a range of only a few tenths of a $\mathrm{MHz}$ and is located below the nuclear Zeeman frequencies in both spectra. The larger part of the silver ENDOR spectrum is isotropic, but in some spectral regions an anisotropic behavior is observed. A similarity of the angular dependences for STE with the angular dependences for the STH, which will be described in Sect. 3, was observed.

The ${ }^{35} \mathrm{Cl}$ and ${ }^{37} \mathrm{Cl}$ ENDOR spectra of the STE show essentially the same features as those of silver; however, the interpretation of the chlorine spectra is more complicated owing to the appearance of additional quadrupole lines.

To describe HF interactions, new terms were added to the general Hamiltonian Eq. (1)

$$
-g_{\mathrm{n}} \beta_{\mathrm{n}} \vec{B} \cdot \vec{I}+\vec{s}_{\mathrm{h}} \cdot \overleftrightarrow{A}_{\mathrm{h}} \cdot \vec{I}+\vec{s}_{\mathrm{e}} \cdot \overleftrightarrow{A}_{\mathrm{e}} \cdot \vec{I}+\vec{I} \cdot \stackrel{\leftrightarrow}{Q} \cdot \vec{I}
$$

Here, the first term describes the Zeeman interaction of a nucleus with spin $\vec{I}$. The $\mathrm{HF}$ interaction of the nucleus with the hole and electron is given by the second and the third terms, respectively. The last term describes the quadrupole interaction (only for $\mathrm{Cl}$ nuclei). The principal values of the $\mathbf{A}$ tensor for the hole are $a_{\mathrm{h} z}, a_{\mathrm{h} x}$ and $a_{\mathrm{hy}}$. The HF interaction for the electron is assumed to be isotropic, which is typical for the HF interaction of a shallow donor, thus $\overleftrightarrow{A}_{\mathrm{e}}=a_{\mathrm{e}}$.

The frequencies for the $\Delta M_{S}=0, \Delta M_{I}= \pm 1$ ENDOR transitions within the triplet sublevels are derived from Eq. (2) and are given by the following expressions [25]:

$$
\text { for } \begin{aligned}
M_{S} & =+1, n_{\mathrm{ENDOR}}=h^{-1}\left|-g_{\mathrm{n}} \beta_{\mathrm{n}} B_{0}+\left(a_{\mathrm{h}}^{\text {eff }}+a_{\mathrm{e}}\right) / 2\right| \\
M_{S} & =-1, n_{\mathrm{ENDOR}}=h^{-1}\left|-g_{\mathrm{n}} \beta_{\mathrm{n}} B_{0}-\left(a_{\mathrm{h}}^{\text {eff }}+a_{\mathrm{e}}\right) / 2\right| \\
M_{S} & =0, n_{\mathrm{ENDOR}}=h^{-1}\left|-g_{\mathrm{n}} \beta_{\mathrm{n}} B_{0}+J^{-1} \beta_{\mathrm{e}} B_{0}\left(g_{\mathrm{h}}^{\text {eff }}-g_{\mathrm{e}}\right)\left(a_{\mathrm{h}}^{\text {eff }}-a_{\mathrm{e}}\right) / 2\right|
\end{aligned}
$$

Here, $g_{\mathrm{h}}^{\text {eff }}=\left(g_{\mathrm{h} z}+g_{\mathrm{h} x}+g_{\mathrm{h} y}\right) / 3$ and $a_{\mathrm{h}}^{\text {eff }}=\left(a_{\mathrm{h} z}+a_{\mathrm{h} x}+a_{\mathrm{h} y}\right) / 3$.

On the basis of the expressions for the ENDOR frequencies, one can qualitatively understand the ENDOR spectra shown in Fig. 4b, which were recorded by monitoring the $M_{S}=0 \leftrightarrow M_{S}=-1$ EPR transition. Since the STE is expected to consist of a delocalized electron trapped in the Coulombic field of a strongly localized hole, hyperfine constants $a_{\mathrm{h}}^{\text {eff }}$ are negligible for the majority of nuclei that contribute to the ENDOR spectrum. According to Eq. (3), the ENDOR transition in the $M_{S}=-1$ level will then lie at the frequency $1 / 2 h^{-1} a_{\mathrm{e}}$ above or below the nuclear Zeeman frequency $h^{-1} g_{\mathrm{n}} \beta_{\mathrm{n}} B_{0}$, depending on the sign of the hyperfine constant $a_{\mathrm{e}}$. For the $M_{S}=0$ level, Eq. (3) predicts a transition at the frequency 
$h^{-1} J^{-1} \beta_{\mathrm{e}} B_{0}\left(g_{\mathrm{h}}^{\text {eff }}-g_{\mathrm{e}}\right) a_{\mathrm{e}} / 2$ above or below the nuclear Zeeman frequency, depending on the signs of $J$ and $a_{\mathrm{e}}$.

The ENDOR spectrum of the $M_{S}=0$ level is compressed by a factor of $\sim 20$, as compared to the spectrum of the $M_{S}=-1$ level. The group of lines covering the broader frequency region in Fig. $4 \mathrm{~b}$ is thus related to ENDOR transitions in the $M_{S}=-1$ level, whereas the compressed group corresponds to transitions in the $M_{S}=0$ level. From the observation that the first group is positioned above the silver Zeeman frequencies and the fact that for silver $g_{\mathrm{n}}$ has a negative sign, we can derive from Eq. (3) that $a_{\mathrm{e}}$ has a negative sign. Because the compressed $M_{S}=0$ ENDOR spectrum in Fig. $4 \mathrm{~b}$ is located below the silver Zeeman frequencies and $a_{\mathrm{e}}<0$, the sign of the exchange energy $J$ has to be negative according to Eq. (3). This is in agreement with previous results. The ENDOR spectrum shown in Fig. 4b, which was recorded by monitoring the $M_{S}=0 \leftrightarrow M_{S}=+1$ EPR transition, confirms this qualitative analysis. Here, both the $M_{S}=+1$ spectrum and the compressed $M_{S}=0$ spectrum are located below the silver Zeeman frequencies, just as predicted by Eq. (3) based on $a_{\mathrm{h}}^{\text {eff }} \approx 0$ and the negative signs of $a_{\mathrm{e}}$ and $J$.

For those nuclei where $a_{\mathrm{h}}^{\text {eff }} \neq 0$, the corresponding ENDOR lines are anisotropic. The dependences were obtained by monitoring the EPR transition of which the resonance field strength $B_{0}$ depends on the orientation of the magnetic field [25]. For a quantitative analysis of the ENDOR spectra, the lattice nuclei were grouped into shells. A shell contains all nuclei that have the same distance $r$ from the central hole.

\subsection{The Spatial Distribution of the Wave Function of STE in $\mathrm{AgCl}$}

The ENDOR study has demonstrated that the lowest triplet state of the STE in $\mathrm{AgCl}$ consists of a very diffuse electron attracted by a strongly localized STH [25]. The spatial distribution of the STE can roughly be divided into three regions. At distances larger than about $18 \AA$, the wave function of the STE is completely determined by the shallow electron, which occupies a hydrogen-like $1 s$ orbital. At distances between 18 and $9 \AA$, the electron is still dominant but its density distribution deviates from the spherical symmetry and reflects the $D_{4 h}$ symmetry of the central STH. At distances shorter than about $9 \AA$, both the central hole and the shallow electron have a contribution to the wave function of the STE. From a comparison with the results of an ENDOR study of the STH, it is concluded that the central hole of the STE is virtually identical to an isolated STH.

For $r>18 \AA$, the ENDOR frequencies are purely determined by HF interaction of the shallow electron and in the case of the $M_{S}= \pm 1$ sublevels are given by $v_{\mathrm{ENDOR}}=h^{-1}\left|-g_{\mathrm{n}} \beta_{\mathrm{n}} B_{0} \pm a_{\mathrm{e}} / 2\right|$. The ENDOR lines for Ag nuclei are isotropic, a property which is typical for the HF interaction between a nucleus and a very delocalized electron. This part of the ENDOR spectrum related to the remote nuclei resembles very much that of the intrinsic shallow electron center in $\mathrm{AgCl}$ which is shown for Ag ENDOR in the bottom of Fig. $4 \mathrm{~b}$ and will be discussed in the next section. The isotropic HF constant which reflects the spin density of the shallow electron wave function $(\Psi)$ at the site of the nucleus $\left(r_{i}\right)$ is given by $a_{i}=(8 \pi / 3) g_{\mathrm{e}} \beta_{\mathrm{e}} g_{\mathrm{ni}} \beta_{\mathrm{n}}\left|\Psi\left(r_{\mathrm{i}}\right)\right|^{2}$, where $g_{\mathrm{ni}}$ is the $g$-factor of nucleus $i$. 
For a quantitative analysis of the observed isotropic HF constants, it is necessary to determine the spin density $\left|\Psi\left(r_{i}\right)\right|^{2}$ on the ligand nucleus. This analysis is based on the prediction of the effective mass theory that the ground state of a shallow donor in semiconductors can be described by a hydrogen-like $1 s$ wave function $\Phi(r) \sim \exp \left(-r / r_{0}\right)$ [28], where $r_{0}$ is effective Bohr radius of the shallow electron center. Since in the STE the shallow electron is bound to a central hole with a Coulombic center at an $\mathrm{Ag}$ ion, we expect that $\Phi(r)$ is centered on an $\mathrm{Ag}$ lattice position. Based on this information, it is possible to assign the lines in the silver ENDOR spectrum to the various shells of the remote class up to the 90th silver shell at a distance of $38.3 \AA$. In the remote region, the electron occupies a spherically symmetrical orbital with an exponential radial dependence, i.e., a hydrogen-like $1 s$ orbital.

At smaller radii in the intermediate and nearby regions, the unpaired electrons' spatial distribution differs in two ways from that in the remote region. First of all, the radial dependence deviates from the exponential behavior. This has been observed before in the case of SECs and was interpreted as the breakdown of the oversimplifying hydrogen model [24]. Second, the angular distribution deviates from the spherical symmetry, thereby causing nuclei within a shell to become inequivalent. One may expect that at small radii, the bound electron becomes sensitive to the charge distribution of the binding core, leading to the redistribution of the electron density. Since the central hole is maintained in a $d\left(x^{2}-y^{2}\right)$-type orbital, its charge distribution possesses $D_{4 h}$ symmetry. Therefore, the angular dependence of the electronic wave function is expected to reflect $D_{4 h}$ symmetry as well. Indeed this seems to be the case as suggested by the characteristic splitting of the intermediate ENDOR lines, and by the assignment of the nearby ENDOR lines. The analysis of the nearby silver nuclei showed that we are able to determine the signs of the HF interactions of the central hole and the diffuse electron. The HF interactions of the hole and the electron were shown to have opposite signs. The HF interaction of the electron with nearby silver nuclei has a negative sign, just as for the remote silver nuclei, which implies that the shallow electron induces a positive electron spin density on silver nuclei (silver nuclei possess a negative gyromagnetic ratio). Similarly, the positive sign of the HF interaction of the hole leads to the conclusion that nearby silver nuclei experience a negative hole spin density. From the remote part of the chlorine ENDOR spectrum, it follows that the shallow electron also induces a positive electron spin density on the chlorine nuclei.

The central hole appears to be strongly localized, and the present results allow us to determine an upper limit of its spatial extension. The spin density of the central hole can only be detected up to the fifth silver shell at a radius of $8.7 \AA$. Since the electron is very diffuse, the overlap of the wave functions of the electron and hole building up the STE is very small, which accounts for the small value of the singlettriplet splitting $J$.

The HF interactions with silver nuclei for the central hole of STE closely resemble those for $\mathrm{STH}$, which will be discussed in detail in Sect. 3. This implies that the electronic structure of the STH is almost identical to that of the central hole of the STE. Apparently, the bound electron is so diffuse that it does not significantly influence the ionic equilibrium positions of the central hole. A small effect of the 
electron can be expected, since the activation energy for STE diffusion is lower than for STH diffusion and, moreover, the presence of the electron is believed to lower the barrier height between the three equivalent configurations of the elongated $\left(\mathrm{AgCl}_{6}\right)^{4-}$ octahedron in the STE compared with that in the STH. In Ref. [21], a tunneling rate of $10^{5} \mathrm{~s}^{-1}$ at $1.2 \mathrm{~K}$ was derived, which should yield a lifetime-limited line width of about $30 \mathrm{kHz}$ for the ENDOR transitions. The fact that in the present study line widths are observed down to $3 \mathrm{kHz}$ suggests that the upper limit of the tunneling rate is rather in the order of $10^{4} \mathrm{~s}^{-1}$.

The similarity between the central hole of the STE and the STH obtained from the ENDOR results indicates that the STH is not accompanied by a nearby vacancy. Indeed, since such an STH-vacancy complex is charge-neutral, one does not expect this complex to attract an electron in a diffuse orbital to form the STE.

Circumstantial experimental evidence exists suggesting that Frenkel pairs are formed in the cationic sublattice of silver halides at liquid-helium temperatures (LHeT) upon UV irradiation. It was proposed that such a pair consists of a shallow electron center at a silver interstitial and an STH center at or near a silver vacancy. Recent experiments suggest that the shallow electron center is in fact formed at a split-interstitial silver pair [24, 26], which is supported by the results of recent Hartree-Fock calculations [29]. In alkali halides, it is well known that Frenkel pairs are created in the anionic sublattice by the nonradiative decay of STEs. In contrast to $\mathrm{AgCl}$ where the STH center resembles a $\left(\mathrm{AgCl}_{6}\right)^{4-}$ molecular ion, the STH center in alkali halides consists of a dimer $X_{2}^{-}$molecular ion, centered between two neighboring anionic positions on the [110] axis (the $\mathrm{V}_{\mathrm{k}}$ center, $X$ represents a halide ion). The $\mathrm{V}_{\mathrm{k}}$ center can capture an electron and form an STE which, after recombination, can produce a Frenkel pair consisting of a molecular $X_{2}^{-}$ionic hole center at a single anionic lattice position (the $\mathrm{H}$ center) and an electron trapped at an anion vacancy (the F center). At LHeT the production of Frenkel pairs occurs most efficiently in those alkali halides where the lowest triplet state of the STE shows offcenter relaxation, i.e., after the attraction of an electron the $X_{2}^{-}$dimer is shifted along the [110] axis. This off-center relaxation is believed to be essential for the Frenkel pair formation at LHeT. In case of the lowest triplet state of the STE in $\mathrm{AgCl}$, no clear evidence for relaxation of the central hole or even of nearby silver ions is observed. Thus, there is no evidence that the suggested production of Frenkel pairs in $\mathrm{AgCl}$ at $\mathrm{LHeT}$ follows a similar path as in alkali halides. Moreover, the lack of observation of STHs accompanied by a vacancy in crystals, where no vacancies are deliberately introduced before irradiation, seems to indicate that the Frenkel pair formation at low temperatures is not efficient in $\mathrm{AgCl}$.

Thus, the spatial distribution of the STE which will be compared with that of the SEC in Sect. 2 can roughly be divided into three regions: remote, intermediate and nearby as shown in Fig. 6b. At distances larger than about $18 \AA$, the wave function of the STE is completely determined by the shallow electron, which occupies a hydrogen-like $1 s$ orbital. At distances between 18 and $9 \AA$, the electron is still dominant but its density distribution deviates from the spherical symmetry and reflects the $D_{4 h}$ symmetry of the central STH. At distances shorter than about $9 \AA$, both the central hole and the shallow electron have a contribution to the wave function of the STE. From a comparison with the results of an ENDOR study of the 
STH, one can conclude that the central hole of the STE is virtually identical to an isolated STH. Since the STE in $\mathrm{AgCl}$ consists of a very diffuse electron attracted by a strongly localized STH, it is of importance to consider separately the SEC and the STH.

\section{Shallow Electron Centers}

In Refs. [24, 26], the first direct reconstruction of the wave function of the intrinsic $\mathrm{SEC}$ in $\mathrm{AgCl}$ and $\mathrm{AgBr}$ was presented and a model of SEC was suggested in which an electron was shallowly trapped by two adjacent silver ions on a single cationic site.

In Fig. 4a the ESE-detected EPR spectrum of the SEC is shown. The ENDOR spectrum of the SEC is presented in Figs. $4 c$ and $5 a-c$. The ENDOR transitions of silver nuclei are shown in Fig. $4 \mathrm{c}$ where the nuclear Zeeman frequencies of ${ }^{107} \mathrm{Ag}$

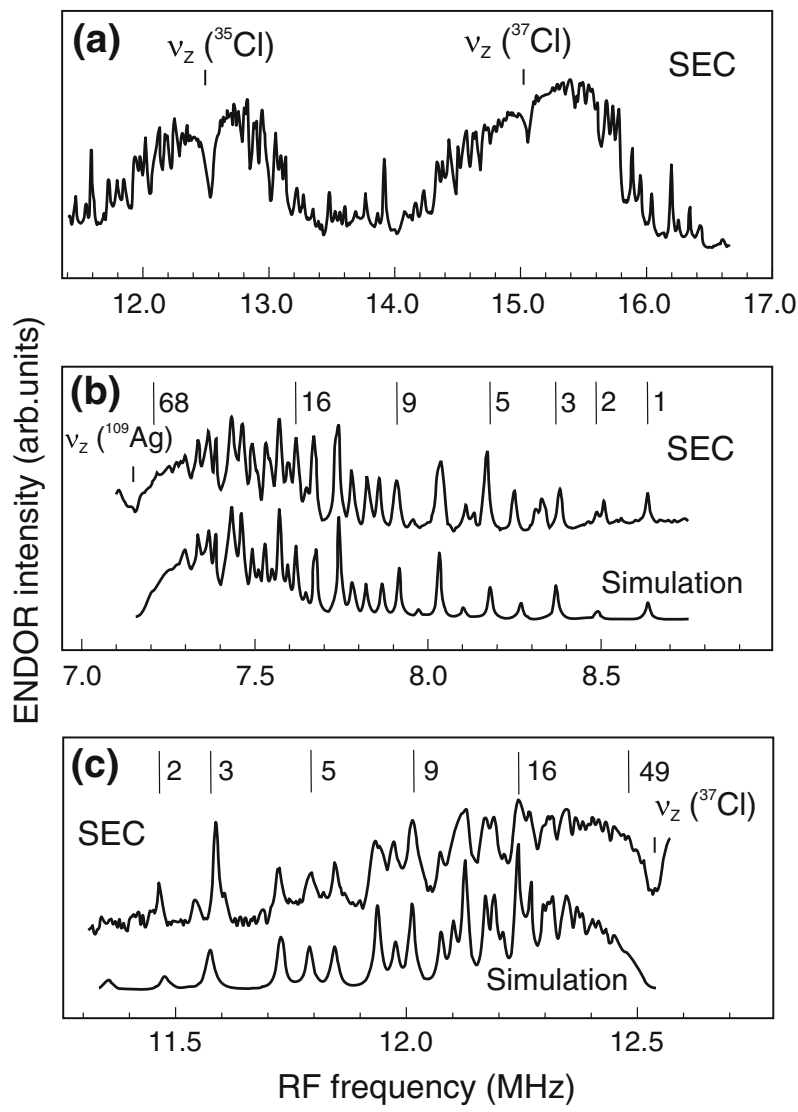

Fig. 5 a Chlorine ENDOR spectra of the shallow electron center $(S=1 / 2, g=1.878)$ recorded under the same conditions as in Fig. 4c. b Comparison between the recorded (upper curve) and simulated (lower curve) high-frequency part of the ${ }^{109} \mathrm{Ag}$ ENDOR spectrum of the SEC. c Similar comparison for the lowfrequency part of the ${ }^{37} \mathrm{Cl}$ ENDOR spectrum. The first $\mathrm{Cl}$ shell is not observed in the ${ }^{37} \mathrm{Cl}$ ENDOR spectrum but is present in the ${ }^{35} \mathrm{Cl}$ spectrum. Lorentzian line shape was used to simulate the ENDOR spectrum 
and ${ }^{109} \mathrm{Ag}$ are observable as dips at 6.224 and $7.156 \mathrm{MHz}$, respectively. Figure 5a shows the chlorine ENDOR transitions and the dips at 12.537 and $15.057 \mathrm{MHz}$ indicate the nuclear Zeeman frequencies of ${ }^{35} \mathrm{Cl}(I=3 / 2,76 \%)$ and ${ }^{37} \mathrm{CI}(I=3 / 2$. $24 \%$ ), respectively. The expanded spectra are shown in Fig. $5 \mathrm{~b}$ and c for the highfrequency part of ${ }^{109} \mathrm{Ag}$ and for the low-frequency part of ${ }^{37} \mathrm{Cl}$, respectively.

The ENDOR spectra proved to be isotropic apart from a few lines in the chlorine spectrum which exhibit a quadrupole splitting. The intensity of these few lines was so weak that the orientational dependences could not be resolved. In case of an electron spin $(S=1 / 2)$ coupled to a single silver nucleus $(I=1 / 2)$, the ENDOR transitions have the following frequencies:

$$
v_{\text {ENDOR }}=h^{-1}\left|g_{\mathrm{n}} \beta_{\mathrm{n}} B_{0} \pm a_{\mathrm{e}} / 2\right| .
$$

For a chlorine nucleus $(I=3 / 2)$, the same expression holds when the quadrupole interaction is neglected. Therefore, Eq. (3) predicts that each nucleus will give rise to two ENDOR transitions symmetrically placed around their nuclear Zeeman frequency. This behavior is indeed observed in the recorded spectra of Figs. 4c and 5. The fact that a multitude of lines is present indicates that we are dealing with a delocalized electron which interacts with a large number of $\mathrm{Ag}$ and $\mathrm{Cl}$ nuclei.

For a quantitative analysis of the HF constants, it is necessary to determine the spin density $\left|\Psi\left(r_{i}\right)\right|^{2}$ on each nucleus $i$. This problem has already been studied for the case of color centers in alkali halides, particularly, the F center. It was shown [30] that by orthogonalizing a suitable envelope function $\Phi(r)$ to the cores of the lattice ions in order to allow for the Pauli principle, the spin density on nucleus $i$ may be written as an "amplification factor" $A_{i}$ times the density of the envelope function $\Phi(r)$ on that nucleus. Thus, $\left|\Psi\left(r_{i}\right)\right|^{2}=A_{i}\left|\Phi\left(r_{i}\right)\right|^{2}$. If the envelope function remains approximately constant within each ion core, the value of $A_{i}$ will only depend on the species of ion $i$ and not on its position in the lattice.

We used a trial-and-error procedure in which we calculated $\left|\Phi\left(r_{i}\right)\right|^{2}$ on a large number of $\mathrm{Ag}$ and $\mathrm{Cl}$ positions in the cubic $\mathrm{AgCl}$ lattice, trying various center positions of $\Phi$ and by optimizing the amplification factor values for $\mathrm{Ag}$ and $\mathrm{Cl}$ and the Bohr radius $r_{0}$ to match the observed HF constants.

The result of our analysis is shown in Fig. 6a where the density of $\Phi$ is plotted as a function of $r$. This result is based on the assumption that $\Phi$ is centered on the $\mathrm{Ag}^{+}$ lattice position. We have been able to derive the density of the envelope function on a large number of $\mathrm{Ag}$ and $\mathrm{Cl}$ shells and some of the $\mathrm{Ag}$ shells are indicated in Fig. 6a. It turned out that for shells with a radius larger than about $12 \AA$, the derived electron densities indeed obey the expected exponential form with a Bohr radius $r_{0}=16.6 \pm 0.8 \AA$ (dashed line in Fig. 6a); however, for nearby shells, there is a clear deviation. This results from the neglect of the influence of the chemical nature of the binding center and it illustrates the need of the so-called central cell correction in the effective mass theory [28]. The derived densities can be very well described by the following normalized monotonically decreasing function [24]

$$
|\Phi(r)|^{2}=\left(7 \pi r_{1}\right)^{-1}\left(1+r / r_{1}\right)^{2} \exp \left(-2 r / r_{1}\right),
$$

which for comparison is plotted as a solid line in Fig. 6a using amplification factors $A_{\mathrm{Ag}}=2,450, A_{\mathrm{Cl}}=1,060$ and $r_{1}=9.94 \AA$. 

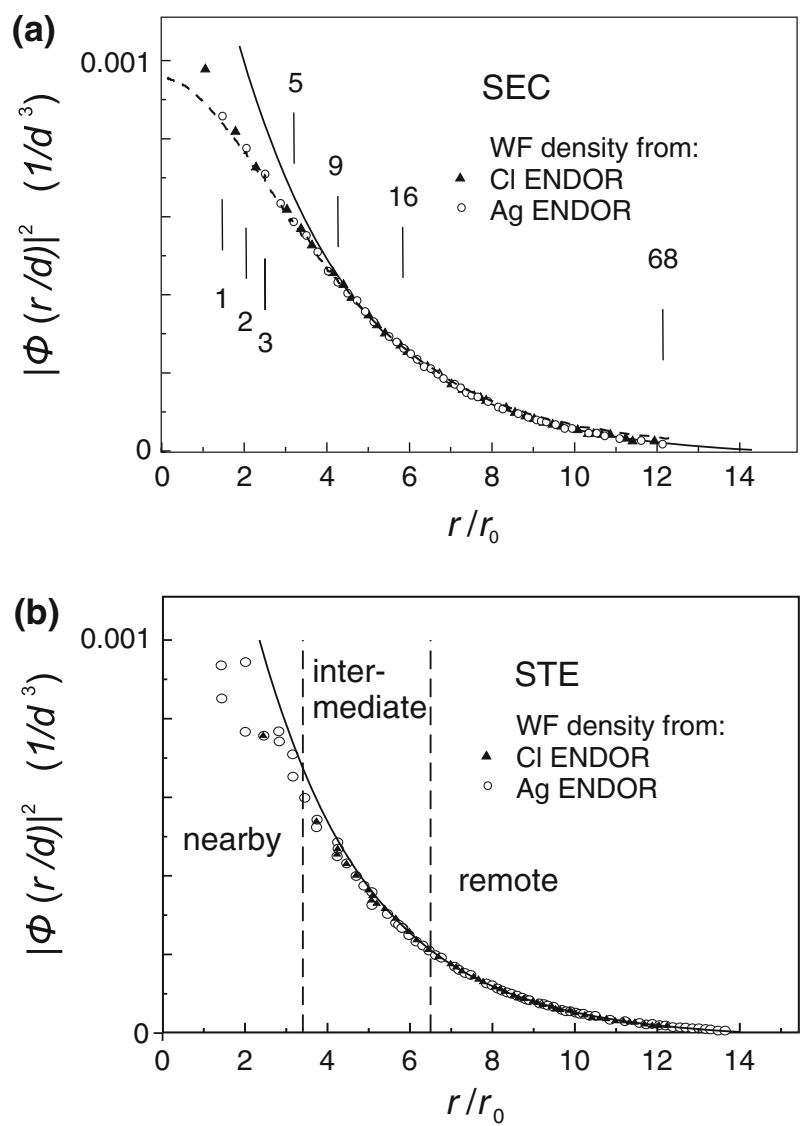

Fig. 6 Density of the envelope $|\Phi(r)|^{2}$ of the SEC (a) and STE (b) as a function of $(r / d)$ with the interionic distance $d=2.753 \mathrm{~A}$. Open circles and triangles denote the densities derived from the $\mathrm{Ag}$ and $\mathrm{Cl}$ ENDOR spectra, respectively. Some neighboring silver shells are indicated. Solid lines illustrate the exponential radial dependence in the remote region corresponding to a Bohr radius $r_{0}=16.6 \AA$ for SEC and $r_{0}=15.1 \AA$ for STE. Dashed line in $\mathbf{d}$ is a result of calculations using Eq. (4)

The experimental ENDOR spectrum was simulated by calculating the frequency of the ENDOR transition using the result of such a simulation of the high-frequency part of the ${ }^{109} \mathrm{Ag}$ ENDOR spectrum shown in Fig. 5b, where it is compared to the recorded spectrum. The figure shows good overall agreement between the recorded and the simulated spectra. The simulation, however, does not account for all features observed at frequencies above 8.1 MHz. The ENDOR lines in this region correspond to the shells that lie close to the center of $\Phi$ and suggest the presence of the lattice distortion in the direct surrounding of the binding core. We indicated the ENDOR transitions of some of the Ag shells. One can see that even the contribution of the 68th silver shell can be resolved. Figure 5c compares the recorded spectrum of the low-frequency part of the ${ }^{37} \mathrm{Cl}$ ENDOR to its corresponding simulation. Again good overall agreement is obtained and the contributions up to the 49th chlorine shell can be observed. 
The results depicted in Fig. $5 \mathrm{~b}$ and $\mathrm{c}$ were obtained by placing the center of the envelope function $\Phi$ on the $\mathrm{Ag}^{+}$lattice position and this turned out to be the only position for which we could obtain a satisfactory analysis of our Ag and $\mathrm{Cl}$ ENDOR spectra. A displacement of the interatomic distance by only $4 \%$ in any direction would already worsen the agreement between the simulated and the recorded spectra.

Information concerning the charge of the intrinsic SEC can be obtained from ENDOR on the STE. The STE consists of an electron loosely bound to an STH and can therefore be considered as a special case of an SEC. It is possible to derive the spatial distribution of the electronic part of the STE, in a similar way as described here for the intrinsic SEC. The density of $\Phi$ for the STE is plotted as a function of $r$ in Fig. 6b. We found that the shallowly trapped electron of the STE also behaves very much like a hydrogen $1 s$ electron, centered on the $\mathrm{Ag}^{+}$lattice position, with a Bohr radius $r_{0}=15.1 \pm 0.6 \AA$ [25]. The close agreement of this value with the one derived for the intrinsic SEC $\left(r_{0}=16.6 \pm 0.8 \AA\right)$ [26] and the fact that the electron of the STE is shallowly bound by the Coulombic field of an STH indicates that the SEC has the same Coulombic charge. For SEC in AgBr, $r_{0}=24.8 \pm 2.3 \AA$ [26].

Since our results show that the intrinsic SEC is located on the $\mathrm{Ag}^{+}$lattice position, the previously suggested model of an interstitial $\mathrm{Ag}^{0}$ "atom", which consists of an electron loosely bound to a single interstitial $\mathrm{Ag}^{+}$ion, was rejected. Such molecular $\mathrm{Ag}_{2}^{+}$ions have been observed in $\mathrm{KCl}$ crystals doped with silver after $\mathrm{X}$-ray irradiation at room temperature; however, in $\mathrm{KCl}$ they form, in contrast to the present situation, deep electron traps [31].

In conclusion, the presented ENDOR results prove that the $g=1.878$ paramagnetic center in undoped $\mathrm{AgCl}$ (previously observed in Ref. [32]) originates from an SEC. Our analysis shows that the center is located on the Ag lattice position within the accuracy of $4 \%$ of the interatomic distance and on the basis of the comparison of the ENDOR results with the optical data [33], we conclude that the center is of intrinsic origin. We suggest that the electron is shallowly trapped in the Coulombic field of two adjacent $\mathrm{Ag}^{+}$ions symmetrically placed on a single cationic site.

\section{Self-Trapped Holes}

In Fig. 7a, the low-temperature ESE-detected EPR spectrum of the STH in $\mathrm{AgCl}$ crystal at $94.9 \mathrm{GHz}$ recorded with the magnetic field oriented along the cubic axis $\langle 100\rangle$ and under continuous UV irradiation is shown [27]. The EPR signals of the STH are indicated in the figure. The transitions labeled with the symbol II belong to the site for which the magnetic field $B_{0}$ is oriented parallel to the distortion $z$-axis, whereas the transitions indicated by $\perp$ result from the two sites with $B_{0}$ perpendicular to this axis. Since the STH complex is Jahn-Teller distorted along one of the cubic axes, the resonances of three sites are observed simultaneously.

To analyze the EPR and subsequent ENDOR spectra of the STH we will use the Hamiltonian

$$
\hat{H}=\beta_{\mathrm{e}} \vec{B} \cdot \overleftrightarrow{g} \cdot \vec{S}+\sum_{i}\left(\vec{S} \cdot \overleftrightarrow{A}_{i} \cdot \vec{I}_{i}+\vec{I}_{i} \cdot \overleftrightarrow{P}_{i} \cdot \vec{I}_{i}-g_{\mathrm{n}} \beta_{\mathrm{n}} \vec{B} \cdot \vec{I}_{i}\right)
$$



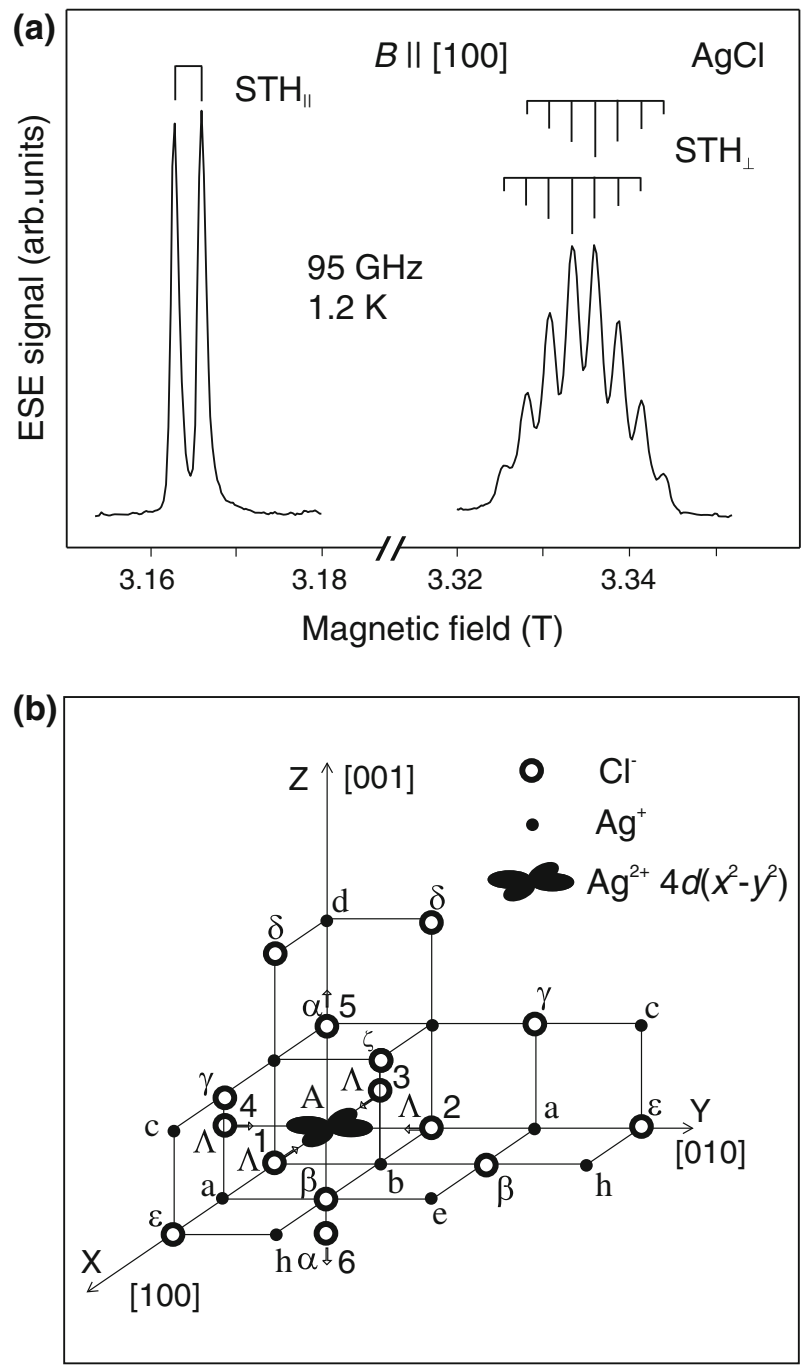

Fig. 7 a ESE-detected EPR spectrum of the STH in $\mathrm{AgCl}$ single crystal recorded under continuous UV irradiation at $94.9 \mathrm{GHz}$ and $1.2 \mathrm{~K}$, with the magnetic field oriented along the $\langle 100\rangle$ cubic axis. b STH in the $\mathrm{AgCl}$ lattice. $X, Y$ and $Z$ are the symmetry axes of the center, $Z$ is taken along the distortion axis. The principal axis system of the HF tensor of the central silver ion is identical to that of the $\mathbf{g}$ tensor. The hole is well localized inside the shells formed by Ag ions labeled as Latin characters A, a, b, c, d, e, h and by $\mathrm{Cl}$ ions labeled as Greek characters $\Lambda, \alpha, \beta, \gamma, \delta, \varepsilon, \zeta$ for which the HF and quadrupole interactions were resolved by ENDOR. The local principal $Z^{\prime}$-axis for the HF tensor of each ligand ion is directed along the line interconnecting the nucleus under consideration and the central silver. The angle $\Theta$ is defined as the angle between the $Z$-axis and the magnetic field, the angle $\varphi$ is defined as the angle between the $X$-axis and the magnetic field that is in the plane perpendicular to the $Z$-axis

Here, $\vec{S}$ represents the electron spin operator of the hole with $S=1 / 2$ and $\vec{I}_{i}$ are nuclear spin operators. The terms with $\vec{I}_{i}$ are summed over the nuclei of $\mathrm{Ag}$ and $\mathrm{Cl}$ that interact with the hole. The $\stackrel{\leftrightarrow}{g}$ tensor reflects the axial symmetry around the 
distortion axis of the STH with principal values $g_{\|}=2.147 \pm 0.002$ and $g_{\perp}=2.040 \pm 0.004$. The second, third and the fourth terms reflect the HF, quadrupole (only for chlorine) and nuclear Zeeman interactions, respectively. The HF interaction parameters can be given in terms of an isotropic part represented by $a$, and a (traceless) anisotropic part represented by $b$ and $b^{\prime}$, which are related to the principal HF-tensor axis system $\left(X^{\prime} Y^{\prime} Z^{\prime}\right)$ by $A_{X^{\prime} X^{\prime}}=a-b+b b^{\prime}, A_{Y^{\prime} Y^{\prime}}=a-b-b^{\prime}$, $A_{Z^{\prime} Z^{\prime}}=a+2 b$. Similarly, we define the diagonal matrix elements describing the nuclear quadrupole interaction in principal axis system as follows: $P_{X^{\prime} X^{\prime}}=$ $-q+q^{\prime}, P_{Y^{\prime} Y^{\prime}}=-q-q^{\prime}, P_{Z^{\prime} Z^{\prime}}=2 q$. The parameters $b^{\prime}$ and $q^{\prime}$ denote the deviation from the axial symmetry. The third term in Eq. (1), which reflects the quadrupole interaction of the chlorine nuclei, can be written in the principal axis system as $P\left[I_{Z^{\prime}}{ }^{2}-(1 / 3) I^{2}\right]+q^{\prime}\left(I_{X^{\prime}}{ }^{2}-I_{Y^{\prime}}{ }^{2}\right)$. Here $P=3 / 2, P_{Z^{\prime} Z^{\prime}}=3 q$ and $q^{\prime}=1 / 2\left(P_{X^{\prime} X^{\prime}}-P_{Y^{\prime} Y^{\prime}}\right)$.

To explain the EPR spectrum of the STH presented in Fig. 7a, it was assumed that only the central silver nucleus and the four equatorial chlorine nuclei in $\langle 100\rangle$ positions in the plane perpendicular to the elongation axis affect the EPR spectrum, since their HF-tensor values are much larger then those of the other nuclei. The HF interactions with other $\mathrm{Ag}$ and $\mathrm{Cl}$ nuclei were not known prior to Refs. [25, 27], and no information was available about quadrupole interactions with the $\mathrm{Cl}$ ions. The surrounding ions of the STH center for which the HF and quadrupole interactions were resolved by EPR and ENDOR are presented in Fig. 7b. The hole is well localized inside the shell formed by Ag ions labeled with Latin characters as A, a, b, $\mathrm{c}, \mathrm{d}, \mathrm{e}, \mathrm{h}$ and by $\mathrm{Cl}$ ions labeled with Greek characters as $\Lambda, \alpha, \beta, \gamma, \delta, \varepsilon, \zeta$. The density outside this shell must be very small. The origin of the axis system coincides with the center of the STH (a silver $\mathrm{Ag}^{2+}$ ion position labeled by $A$ ). The following orientations for the principal axes system $X^{\prime}, Y^{\prime}, Z^{\prime}$ of the equatorial chlorine HFtensor with respect to the distortion axes $X, Y$ and $Z$ of the STH shown in Fig. 7b, were used: the $Z^{\prime}$ principal axis of the HF-tensor is directed along the line interconnecting the chlorine nuclei 1, 2, 3 and 4 and the central silver, $X^{\prime}$ lies in the $X Y$ plane and $Y^{\prime}$ is perpendicular to the $X Y$ plane.

The doublet structure of the $\mathrm{STH}_{\|}$transition in Fig. 7a indicates that when $B_{0}$ is oriented along the distortion axis, the HF coupling with the central silver nucleus is dominant compared to the HF couplings with four equatorial chlorine ligands labeled as $\mathrm{Cl}_{\Lambda}$, and $\mathrm{HF}$ couplings with other surrounding nuclei. From the observed splitting, a value of $A_{\|}$was previously derived (Table 2). In the case of the $\mathrm{STH}_{\perp}$ transition, where $B_{0}$ is oriented perpendicular to the distortion axis, the observed eightfold structure results from the dominant HF interaction of the hole with the central silver nucleus and with the chlorine nuclei of two equivalent equatorial chlorine ligands, for which the HF-interaction parameters were previously derived and presented in Table 2.

From the ENDOR experiment, the HF and quadrupole interactions with ligands that are not resolved in EPR were obtained. Also, much more accurate values for the central $\mathrm{Ag}_{\mathrm{A}}$ and equatorial $\mathrm{Cl}_{\Lambda} \mathrm{HF}$ interactions could be measured. Figure 8 shows the low-frequency part (Fig. 8a) and the high-frequency part (Fig. 8b and c) of the ENDOR spectra recorded with the magnetic field oriented along the $Z$ (Fig. 8b) and $X$ (Fig. 8c) axes of the g-tensor by monitoring the EPR transitions $\mathrm{STH}_{\|}$and $\mathrm{STH}_{\perp}$ 
Table $2 \mathrm{HF}$ interaction parameters of the central silver $\left({ }^{109} \mathrm{Ag}_{\mathrm{A}}\right)$ and $\mathrm{HF}$ and quadrupole interaction parameters of the equatorial chlorine ions $\left(\mathrm{Cl}_{\Lambda}\right)$ of the $\mathrm{STH}$

\begin{tabular}{llllllll}
\hline $\begin{array}{l}\text { Central } \\
\text { silver } \\
\left(\mathrm{Ag}_{\mathrm{A}}\right)\end{array}$ & $\begin{array}{l}A_{\|} \\
(\mathrm{MHz})\end{array}$ & $\begin{array}{l}A_{\perp} \\
(\mathrm{MHz})\end{array}$ & $\begin{array}{l}\text { Equatorial } \\
\text { chlorine ions }\left(\mathrm{Cl}_{\Lambda}\right)\end{array}$ & $\begin{array}{l}A_{Z^{\prime} Z^{\prime}} \\
(\mathrm{MHz})\end{array}$ & $\begin{array}{l}A_{Y^{\prime} Y^{\prime}} \\
(\mathrm{MHz})\end{array}$ & $\begin{array}{l}2|P| \\
(\mathrm{MHz})\end{array}$ & $\begin{array}{l}\text { Ref. } \\
\end{array}$ \\
\hline${ }^{109} \mathrm{Ag}$ & $(-) 100.0(1)$ & $(-) 65.8(1)$ & ${ }^{35} \mathrm{Cl}$ & $(-) 81.4(1)$ & $(+) 6.4(1)$ & $10.3(1)$ & {$[27]$} \\
${ }^{109} \mathrm{Ag}$ & $96 \pm 6$ & $63 \pm 6$ & & $86 \pm 6$ & & {$[7-9]$}
\end{tabular}

Only the absolute value has been obtained for the quadrupole interaction. The uncertainty in the last digit of these constants is indicated in brackets. The signs of the HF interactions (in brackets) were determined from the 'anomalous' ENDOR effect [27]. Note that in Ref. [21] only absolute values of the HF parameters were obtained

shown in Fig. 7a. The frequencies of the ENDOR transitions in these spectra can be analyzed by the first-order solution of the Hamiltonian Eq. (5), with the selection rules $\Delta m_{S}=0, \Delta m_{I}= \pm 1$, which yields

$$
v_{\mathrm{ENDOR}}\left(m_{S}\right)=(1 / h)\left|A m_{S}-g_{\mathrm{n}} \beta_{\mathrm{n}} B_{0}+m_{q}(2 P)\right|,
$$

where $A$ is the HF coupling with the central silver $\left(\mathrm{Ag}_{\mathrm{A}}\right)$. The HF interaction of the four equatorial chlorine ligands are labeled as $\mathrm{Cl}_{\Lambda}$ and the $\mathrm{HF}$ interactions for remote silver and chlorine nuclei are labeled by a to h and $\alpha$ to $\zeta$, respectively. $P$ is the quadrupole interaction parameter for chlorine nuclei and the variable $m_{q}$ is given by $m_{q}=1 / 2\left(m_{I}+m_{I}^{*}\right)$, where $m_{I}$ and $m_{I}^{*}$ are the chlorine nuclear spin states involved in the transition. For the central silver, $\left|A\left(\mathrm{Ag}_{\mathrm{A}}\right)\right| \gg\left|g_{\mathrm{n}}(\mathrm{Ag}) \beta_{\mathrm{n}} B_{0}\right|$ and it is clear from Eq. (5) that for each silver isotope, two transitions are expected which must be separated by two times the nuclear Zeeman frequency $v_{\mathrm{Z}}(\mathrm{Ag})=\lg _{\mathrm{n}}(\mathrm{Ag})$ $\beta_{\mathrm{n}} B_{0} \mathrm{l}$. These transitions are easily recognized in the spectra of Fig. $8 \mathrm{~b}$ and $\mathrm{c}$, and the extracted values are listed in Table 2 . These values are in agreement with the ones found by EPR, which are less accurate and moreover reflect the averaged value of ${ }^{107} \mathrm{Ag}$ and ${ }^{109} \mathrm{Ag}$ interactions.

The HF couplings with chlorine isotope of the four equatorial chlorine ligands $\left(\mathrm{Cl}_{\Lambda}\right)$ are in the limit that $\left|A\left(\mathrm{Cl}_{\Lambda}\right)\right| \gg\left|g_{\mathrm{n}}(\mathrm{Cl}) \beta_{\mathrm{n}} B_{0}\right|$. This again leads to the detection of two lines with a separation of two times the nuclear Zeeman frequency $v_{Z}(\mathrm{Cl})=\mid g_{\mathrm{n}}(\mathrm{Cl}) \beta_{\mathrm{n}} B_{0} l$, corresponding to the transitions for which $m_{q}=0$. However, each $m_{q}=0$ 'HF' line is accompanied by two additional lines at $\Delta v= \pm 2 P$, which correspond to $m_{q}= \pm 1$ transitions. In Fig. 8c we have marked all six ENDOR transitions of the ${ }^{37} \mathrm{Cl}$ and ${ }^{35} \mathrm{Cl}$ isotopes and attached the $m_{q}$ value in the subscript. The corresponding HF interactions $A_{Z^{\prime} Z^{\prime}}\left(\mathrm{Cl}_{\Lambda}\right)$ are listed in Table 2 and agree with those obtained from EPR, which are again less accurate and reflect the averaged value of ${ }^{35} \mathrm{Cl}$ and ${ }^{37} \mathrm{Cl}$ interactions. Also, the magnitude of the quadrupole interaction $P_{Z^{\prime} Z^{\prime}}\left(\mathrm{Cl}_{\Lambda}\right)$ is included in Table 2. Figure $8 \mathrm{c}$ is recorded with $B_{0} \| X$ and according to the definition of the principal axes of the HF tensor in Fig. 8b, the derived values apply for $\mathrm{Cl}_{\Lambda}$ ligands 1 and 3 . For this orientation, $\mathrm{Cl}_{\Lambda}$ ligands 2 and 4 should give rise to resonances from which the value of $A_{X^{\prime} X^{\prime}}\left(\mathrm{Cl}_{\Lambda}\right)$ can be obtained. Since the EPR spectrum already indicates that the magnitude of $A_{X^{\prime} X^{\prime}}\left(\mathrm{Cl}_{\Lambda}\right)$ is small, the resonances of ligands 2 and 4 will probably lie close to or even coincide with 
(a)
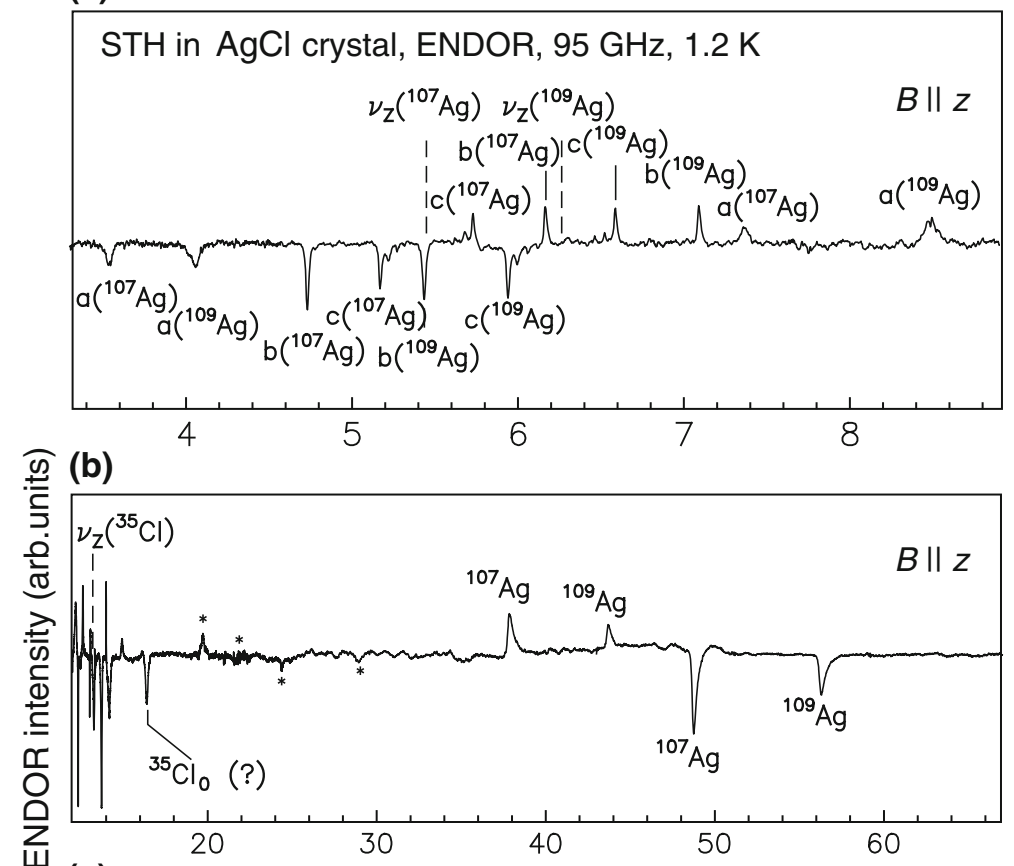

(c)

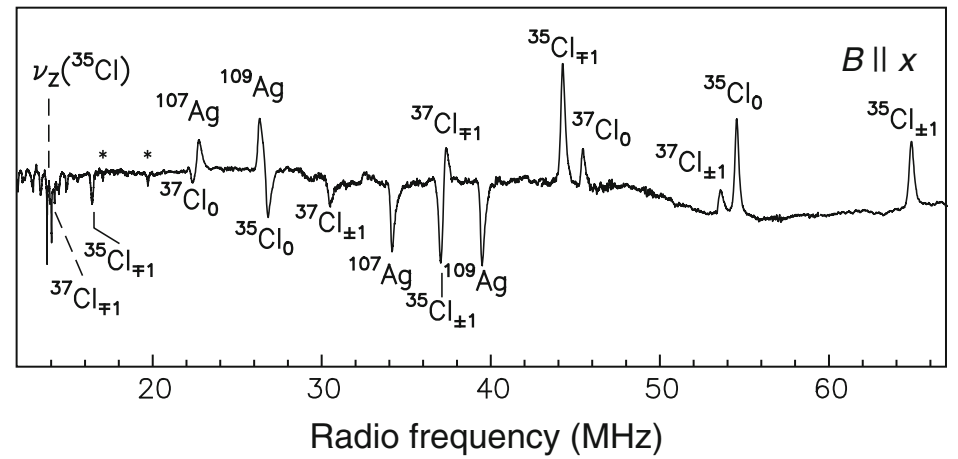

Fig. 8 Low-frequency (a) and high-frequency (b) parts of the ENDOR spectra of the STH recorded with $B_{0} \| Z$, and high-frequency part (c) recorded with $B_{0} \| X$ by monitoring the EPR transitions indicated in Fig. 7. The ${ }^{107} \mathrm{Ag}$ and ${ }^{109} \mathrm{Ag}$ resonances of the central silver ion and the ${ }^{37} \mathrm{Cl}$ and ${ }^{35} \mathrm{Cl}$ resonances of the four equatorial chlorine ligands are indicated. In a $v_{Z}\left({ }^{107} \mathrm{Ag}\right)=5.4 \mathrm{MHz}, v_{Z}\left({ }^{109} \mathrm{Ag}\right)=6.3 \mathrm{MHz}$ and $v_{\mathrm{Z}}\left({ }^{35} \mathrm{Cl}\right)=13.2 \mathrm{MHz}$, whereas in $\mathbf{b} v_{\mathrm{Z}}\left({ }^{107} \mathrm{Ag}\right)=5.7 \mathrm{MHz}, v_{\mathrm{Z}}\left({ }^{109} \mathrm{Ag}\right)=6.6 \mathrm{MHz}, v_{\mathrm{Z}}\left({ }^{37} \mathrm{Cl}\right)=11.6 \mathrm{MHz}$ and $v_{\mathrm{Z}}\left({ }^{35} \mathrm{Cl}\right)=13.9 \mathrm{MHz}$. The transitions marked with asterisks are higher harmonics of the central silver ENDOR transition due to nonlinearity of the radio-frequency amplifier

those of the surrounding chlorine neighbors. In principle, the low-frequency $\mathrm{Cl}_{\Lambda} \mathrm{HF}$ resonances might be identified from the angular dependence of the high-frequency ENDOR lines of Fig. 8c, when rotating the magnetic field $B_{0}$ from the $X$ - to the $Y$ axis. Unfortunately, when $B_{0}$ is rotated over more than $10^{\circ}$ from $X$, the highfrequency $\mathrm{Cl}_{\Lambda} \mathrm{HF}$ resonances disappear. A similar observation is made when $B_{0}$ is 
rotated from $X$ to $Z$, and consequently it is not immediately clear where the $\mathrm{Cl}_{\Lambda} \mathrm{HF}$ transitions are positioned for $B_{0} \| Z$. The only candidate for the $\mathrm{Cl}_{\Lambda} \mathrm{HF}$-transition in this latter orientation, which is left after most of the chlorine HF-lines have been assigned, is the line at $16.4 \mathrm{MHz}$ in Fig. 8b. If we assume that this line corresponds to the $m_{q}=0$ ' $\mathrm{HF}$ ' transition, this yields $\left|A_{Y^{\prime} Y^{\prime}}\left({ }^{35} \mathrm{Cl}_{\Lambda}\right)\right|=6.4 \pm 0.1 \mathrm{MHz}$. This value is in reasonable agreement with the one estimated from the EPR line width of the $\mathrm{STH}_{\|}$transition $\left|A_{Y^{\prime} Y^{\prime}}\left({ }^{35} \mathrm{Cl}_{\Lambda}\right)\right|=5.7 \mathrm{MHz}$.

Figures $8 \mathrm{a}$ and 9 show the low-frequency part (with $v_{\mathrm{RF}}<15 \mathrm{MHz}$ ) of the ENDOR spectrum recorded with $B_{0}$ parallel to the distortion axis of the STH, which contains the silver and chlorine HF-transitions. These spectra can be interpreted by Eq. (6) in the limit that the nuclear Zeeman frequencies of the silver and chlorine isotopes are large. Thus, two transitions are expected for each isotope at a distance $A / 2$ above and below $v_{\mathrm{Z}}$, which indeed is the case as indicated by the labels in Fig. 9a. For the $m_{q}=0$ 'hyperfine' transitions of the chlorine isotopes, a similar distribution is valid though the presence of the $m_{q}= \pm 1$ 'quadrupole' lines, which makes the chlorine spectrum of Fig. 9 less transparent.

The interaction parameters of the silver and chlorine HF-transitions, which are shown in Figs. 8a and 9, can be extracted from the angular dependence of the line positions. Figure $9 \mathrm{~b}$ presents the angular dependence of the high-frequency part of the ${ }^{35} \mathrm{Cl}$ ENDOR spectrum (indicated by asterisks). Here, the nuclear Zeeman frequency of the ${ }^{35} \mathrm{Cl}$ nucleus has been subtracted to remove the frequency shift caused by the anisotropy of the g-tensor. The results of the analysis of the silver HF interaction are presented in Table 2. Most of the HF tensors are assigned to nuclei assuming that the $Z^{\prime}$ principal axis of the tensor is directed along the line interconnecting the central silver ion and the involved nucleus. The listed values have been obtained from the best fit of the ENDOR angular dependences using the computer package "Visual EPR" by Grachev [34].

The analysis of the chlorine ENDOR angular dependence is more complicated than the analysis of the Ag ENDOR spectra due to the presence of the $m_{q}= \pm 1$ 'quadrupole' lines. Still a few characteristic patterns can be recognized, as indicated by the solid lines in Fig. 9b, and the HF and quadrupole parameters resulting from the fit of these dependences can be extracted (see Table 3). The obtained HF parameters are split into the isotropic and anisotropic parts. The assignment of the lines labeled with Greek characters in the spectra of Figs. 8 and 9 is based on a simplifying assumption that the $Z^{\prime}$ principal axis of both the HF and quadrupole tensors is directed along the line interconnecting the central silver ion and the respective chlorine neighbor. Note that the chlorine HF results should be treated with some caution owing to the many overlapping ENDOR angular dependences in Fig. 9b. This especially applies for lines ' $\beta$ ' and ' $\gamma$ ', which can only be recognized in the $X Y$ plane and for which the derived parameters are based on fits that include a small number of points. For simplicity, the angular dependences of the lines ' $\beta$ ' and ' $\gamma$ ' are not included in Fig. $9 \mathrm{~b}$.

In general, the analysis of the ENDOR angular dependences only yields the relative signs of the HF parameters $a, b$, and $b^{\prime}$. For the silver HF interaction, the absolute signs can be obtained from the ENDOR study of the lowest triplet state of the STE in $\mathrm{AgCl}$ [26]. It is well established that the STE consists of an electron, 

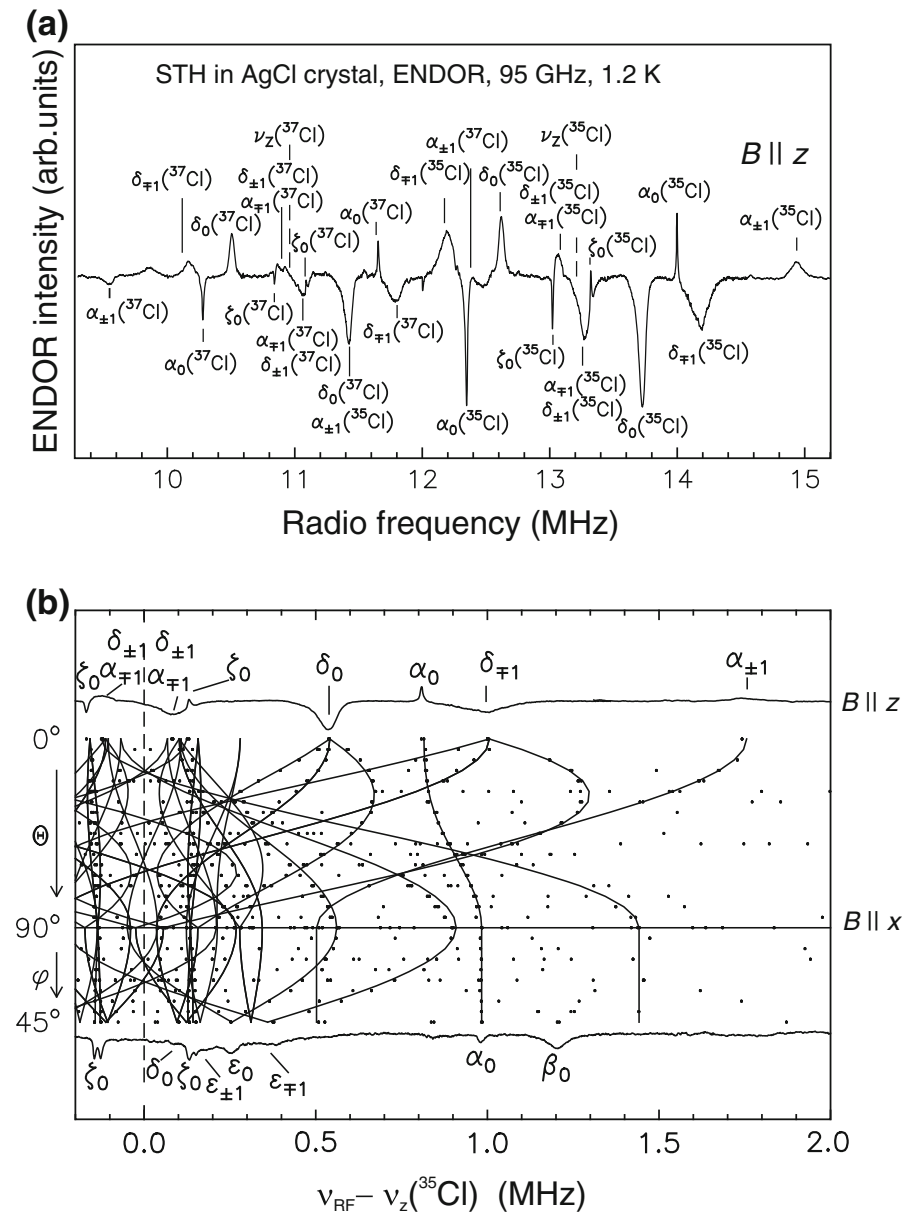

Fig. 9 a Low-frequency part of the ENDOR spectra of the chlorine HF resonances recorded with $B_{0} \| \mathrm{Z}$. b Angular dependence of the high-frequency part of the ${ }^{35} \mathrm{Cl}$ ENDOR spectrum. The nuclear Zeeman frequency of ${ }^{35} \mathrm{Cl}$ has been subtracted to eliminate the frequency shift induced by the anisotropy of the gtensor

which is shallowly trapped in the Coulombic field of an STH, and both the electron and the hole contribute to the observed ENDOR frequencies. The absolute signs of HF coupling for the STH complex are included in Table 4. Unfortunately, the absolute signs of the central silver and chlorine HF interactions cannot be verified from the study of the STE because the ENDOR resonances of the central silver and the equatorial chlorines were not observed for the STE. Since it is questionable whether the chlorine angular dependences in the very dense spectra of the more complex STE system would be resolved and because the recording of such dependences is very time-consuming, no attempt was undertaken to do so.

The spin density distribution of the STH complex in $\mathrm{AgCl}$ was found by using one-electron wave function of the unpaired electron bound to the STH which was constructed as a linear combination of atomic orbitals (LCAO) centered on silver 


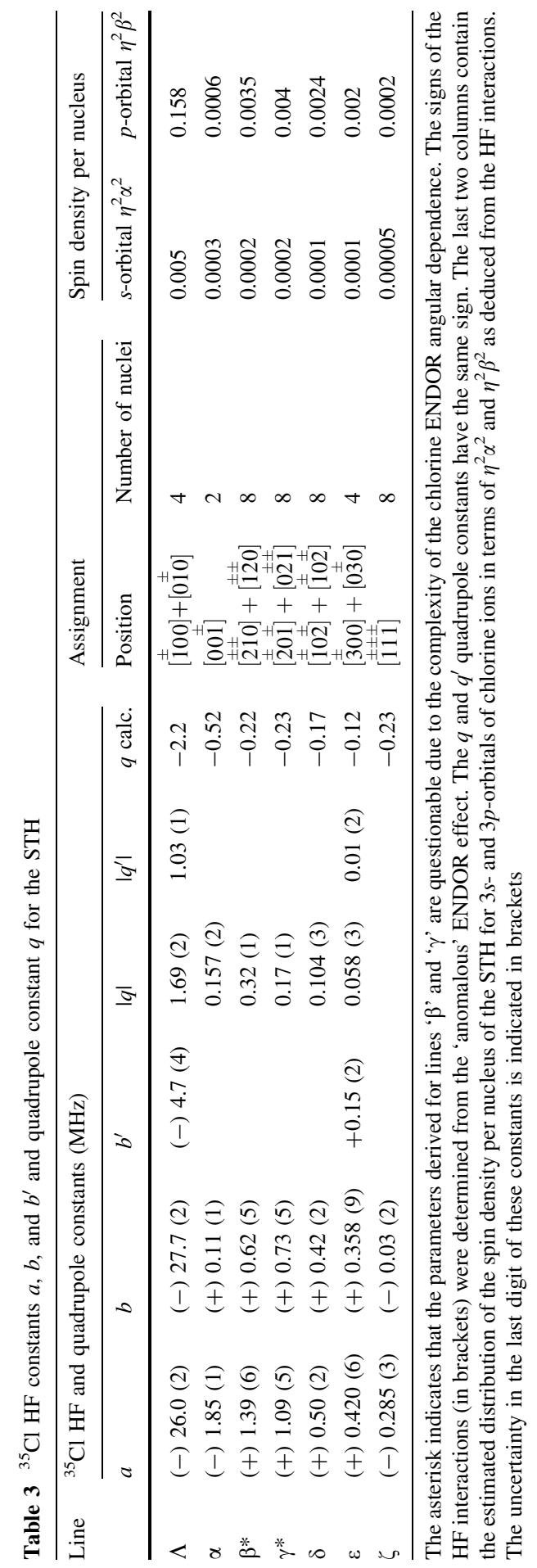


Table $4{ }^{109} \mathrm{Ag} \mathrm{HF}$ constants $a, b$, and $b^{\prime}$ for the STH

\begin{tabular}{|c|c|c|c|c|c|c|c|}
\hline \multirow[t]{2}{*}{ Line } & \multicolumn{3}{|c|}{${ }^{109} \mathrm{Ag} \mathrm{HF}$ constants } & \multicolumn{2}{|l|}{ Assignment } & \multicolumn{2}{|c|}{ Spin density per nucleus } \\
\hline & $\begin{array}{l}a \\
(\mathrm{MHz})\end{array}$ & $\begin{array}{l}b \\
(\mathrm{MHz})\end{array}$ & $\begin{array}{l}b^{\prime} \\
(\mathrm{MHz})\end{array}$ & Position & $\begin{array}{l}\text { Number } \\
\text { of nuclei }\end{array}$ & $\begin{array}{l}s \text {-orbital } \\
\eta^{2} \alpha^{2}\end{array}$ & $\begin{array}{l}d \text {-orbital } \\
\eta^{2} \beta^{2}\end{array}$ \\
\hline A & $(-) 77.2(4)$ & $(-) 11.3(2)$ & & Central & 1 & 0.041 & 0.193 \\
\hline $\mathrm{a}$ & $+3.02(1)$ & $-0.81(1)$ & & {$[\stackrel{ \pm}{2} 00]+[0 \stackrel{ \pm}{2} 0]$} & 4 & 0.0016 & 0.014 \\
\hline $\mathrm{b}$ & $+1.396(3)$ & -0.033 & -0.014 & {$[110]$} & 4 & 0.0008 & 0.0006 \\
\hline $\mathrm{c}$ & $+0.531(3)$ & $-0.030(3)$ & $0.013(5)$ & {$\left[\begin{array}{l} \pm \\
0\end{array} \frac{ \pm}{1}\right]+\left[03 \frac{ \pm}{1}\right]$} & 8 & 0.0003 & 0.0005 \\
\hline $\mathrm{d}$ & $+0.364(3)$ & $+0.050(3)$ & & {$[00 \stackrel{ \pm}{2}]$} & 2 & 0.0002 & 0.0009 \\
\hline $\mathrm{e}$ & $+0.329(3)$ & $-0.011(3)$ & $-0.018(3)$ & {$[220]$} & 4 & 0.0002 & 0.0002 \\
\hline $\mathrm{h}$ & $+0.10(3)$ & $0.01(3)$ & $0.01(3)$ & {$\left[\begin{array}{l} \pm \pm \\
310\end{array}\right]+\left[\begin{array}{l} \pm \pm \\
130\end{array}\right]$} & 8 & 0.00005 & 0.0001 \\
\hline
\end{tabular}

The signs were obtained from the ENDOR study of the lowest triplet state of the STE in AgCl. The signs of the HF interaction for the central silver $\mathrm{Ag}_{\mathrm{A}}$ (in brackets) was determined from the 'anomalous' ENDOR effect. The last two columns contain the estimated distribution of the spin density per nucleus of the STH for $s$ - and $d$-orbitals of silver ions in terms of $\eta^{2} \alpha^{2}$ and $\eta^{2} \beta^{2}$ as deduced from the HF interactions. A possible contribution from the core polarization and point dipole-dipole interaction was not considered. The uncertainty in the last digit is indicated in brackets

and chlorine sites in the vicinity of the STH in line with theoretical calculations [35, 36]. It was shown that the wave function of STH is mainly distributed over five nuclei and not only located on the central nucleus. About $19 \%$ of the spin density is located in the $4 d\left(x^{2}-y^{2}\right)$ orbital on the central silver $\mathrm{Ag}_{\mathrm{A}}$, and about $65 \%$ of the spin density is located in the $3 s$ and $3 p$ orbitals of the four equatorial chlorines of $\mathrm{Cl}_{\Lambda}$. The remainder of the wave function $(\sim 16 \%)$ is spread mostly over the chlorine and the silver shells that are mainly near the $X Y$ plane perpendicular to the elongation axis. This includes up to $7 \%$ of the spin density which is localized on the ions situated at the $X$ and $Y$ axes outside the central square-planar $\left(\mathrm{AgCl}_{4}\right)^{2-}$ unit. Thus, the hole is essentially located in the plane perpendicular to the elongation axis. In general, our results show that the hole is distributed on $\mathrm{Ag}(\sim 30 \%)$ and $\mathrm{Cl}(\sim 70 \%)$ sublattices and this finding confirms the $4 d\left(\mathrm{Ag}^{+}\right)$and $3 p\left(\mathrm{Cl}^{-}\right)$orbital admixture at the maximum of the valence band.

The quadrupole interactions have been determined from the ENDOR analysis that gave the electrical field gradient distribution at the chlorine sites. It was shown that the largest quadrupole interactions were observed near the plane perpendicular to the elongation axis and that they correlate with the values of the anisotropic HF interactions. The large deviation from the axial symmetry is found for quadrupole interactions with the four equatorial chlorine ions and for interaction with chlorine ions that are positioned along the $X$ and $Y$ axes outside of the central square-planar $\left(\mathrm{AgCl}_{4}\right)^{2-}$ unit.

The results of the low-temperature ENDOR study of the STH complex in $\mathrm{AgCl}$ confirm that the trapping process is of intrinsic nature, i.e., a Jahn-Teller distortion is not accompanied by a charged vacancy or impurity. This conclusion is based on the finding that the ENDOR angular dependences of neighboring silver and chlorine nuclei still reflect the $D_{4 h}$ local symmetry. This eliminates the possible involvement of an uncharged impurity or intrinsic defect unless it is located at a distant position 
for which the role in the trapping process can only be of secondary importance. The results of calculations of the quadrupole interactions are also in line with the suggestion that the $\mathrm{STH}$ complex in $\mathrm{AgCl}$ is of intrinsic nature.

It was previously accepted that no self-localization of holes takes place in the bulk $\mathrm{AgBr}$ (see, for example, Refs. [2, 16]). However, one cannot exclude that the holes in $\mathrm{AgBr}$ can be self-localized as well. In contrast to the situation in $\mathrm{AgCl}$, the dynamic Jahn-Teller effect may take place in $\mathrm{AgBr}$, which leads to isotropic $g$ value, as it is observed in experiment. The $g$ value of holes in $\mathrm{AgBr} g=2.08$ is close to an average $g$ value of the STH in $\mathrm{AgCl}$. According to this approach, the bound exciton in $\mathrm{AgBr}$ may possess qualitatively the same structure as the STE in $\mathrm{AgCl}$ in which the wave function of an electron trapped by an STH is close to the wave function of a SEC. A smaller magnitude of the singlet-triplet splitting observed in $\mathrm{AgBr}$ could be explained by a more strongly delocalized wave function of the electron part of the exciton.

\section{Silver Halide Micro- and Nanocrystals Embedded in Alkali Halide Crystalline Matrix}

\section{1 $\mathrm{AgCl}$ Micro- and Nanocrystals in $\mathrm{KCl}$}

The effects of confinement on shallow centers with the Bohr radius comparable with the particle size are well known. The influence of the nanoparticle size on deep level centers and local effects in solids is of fundamental importance, however, it is much less studied to our knowledge. The Jahn-Teller effect is one of the basic local effects in solids, rather sensitive to internal fields and variations of the electronphonon interaction. The STH and STE in bulk $\mathrm{AgCl}$ are classical Jahn-Teller systems, well studied by high-frequency ESE and ENDOR as was shown above. Therefore, investigation of $\mathrm{AgCl}$ nanocrystals embedded in a crystalline matrix seems to be very promising.

Self-organized growth of silver halide micron-size and nanometer-size crystals (microcrystals and nanocrystals) retaining the orientation of the host lattice was found in $\mathrm{KCl}: \mathrm{Ag}$ and $\mathrm{KBr}: \mathrm{Ag}$ crystals grown from melt with $1-3 \%$ of silver impurity [37-42]. Silver halide $\mathrm{AgCl}$ and $\mathrm{AgBr}$ crystals have the same face-centered cubic lattice as alkali halides $\mathrm{KCl}$ and $\mathrm{KBr}$ and slightly different lattice constants, but the energy gaps in silver halides are more than $5 \mathrm{eV}$ larger. Thus, nanometersize $\mathrm{AgCl}$ and $\mathrm{AgBr}$ crystals embedded in $\mathrm{KCl}$ and $\mathrm{KBr}$ matrices can be considered as an array of self-organized nanocrystals (quantum dots) in a strained heterosystem.

It was unambiguously shown by ODMR that silver halide microcrystals embedded in alkali halide crystals retain the properties of bulk silver halides since the ODMR spectra of the microcrystals were practically the same as those in bulk $\mathrm{AgCl}$ and $\mathrm{AgBr}$. The characteristic ODMR spectra of the STH, STE and SEC in bulk $\mathrm{AgCl}$ were used as a 'fingerprint' for the embedded $\mathrm{AgCl}$ microcrystals in $\mathrm{KCl}$ and as a proof of their crystallinity [23, 38, 40]. Figure 10 shows the photoluminescence (PL) (Fig. 10a) and the $35.2 \mathrm{GHz}$ ODMR (Fig. 10b) spectra recorded at $1.8 \mathrm{~K}$ in two samples which were cleaved from different parts of the $\mathrm{KCl}: \mathrm{AgCl}$ 

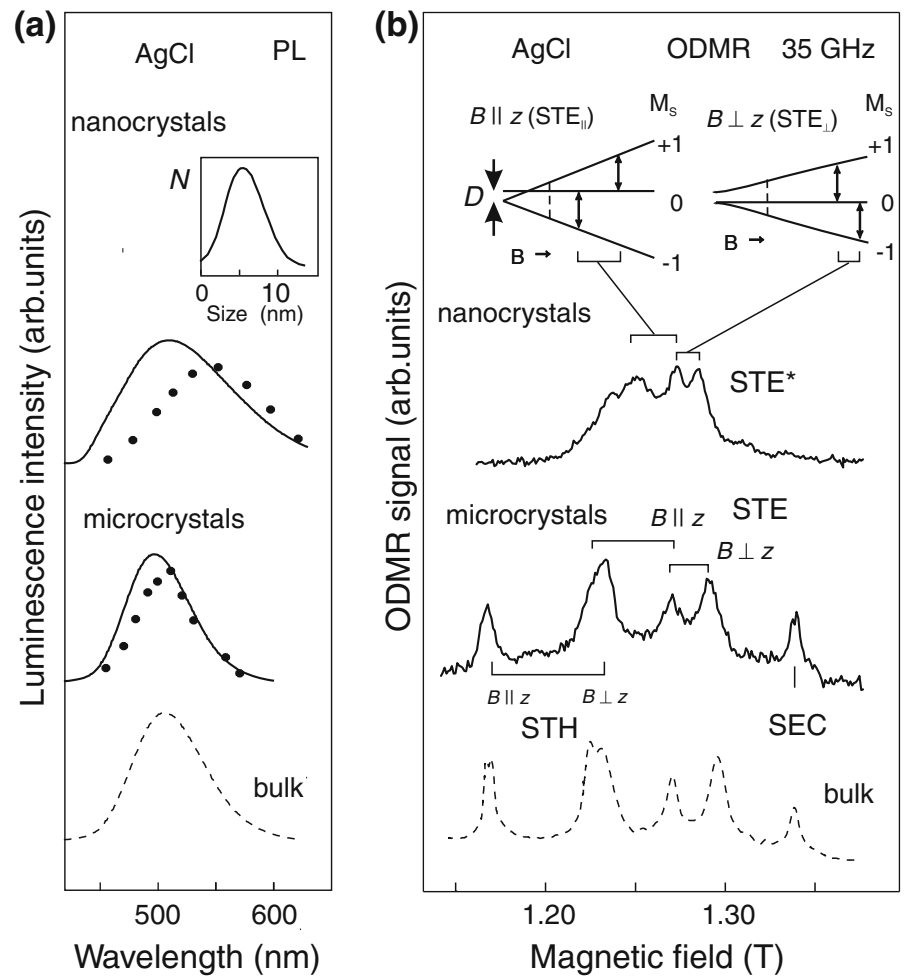

Fig. 10 a $\mathrm{PL}$ and b ODMR spectra in $\mathrm{AgCl}$ microcrystals and nanocrystals embedded in the $\mathrm{KCl}$ crystalline matrix. For comparison the luminescence and ODMR spectra in bulk $\mathrm{AgCl}$ are shown by broken curves. Points in a show the spectral dependences of the ODMR signals. The positions of the ODMR lines are marked for the STE, STH and SEC in $\mathrm{AgCl}$ microcrystals and STE* in $\mathrm{AgCl}$ nanocrystals. Symbols $\|$ and $\perp$ denote the centers with their axes parallel and perpendicular to the magnetic field, respectively. $T=1.8 \mathrm{~K} ; v=35.2 \mathrm{GHz} ; P=300 \mathrm{~mW} ; f_{\mathrm{m}}=80 \mathrm{~Hz} ; B \|[001]$. The energy level diagrams for the triplet state of the STE* and the observed EPR transitions are shown at the top of $\mathbf{b}$. Inset in a shows the size distribution of the $\mathrm{AgCl}$ nanocrystals based on the data from Ref. [37]

crystal (2\% $\mathrm{AgCl}$ in $\mathrm{KCl}$ melt) and contained $\mathrm{AgCl}$ microcrystals (sample 1) and nanocrystals (sample 2). The spectra of bulk $\mathrm{AgCl}$ crystals, measured under the same experimental conditions, are shown for comparison by broken curves. The energy levels with the observed transitions for the triplet state of the STE are shown at the top of Fig. 10b. Points in Fig. 10a represent the spectral dependences of the ODMR amplitude for the STE. A remarkable similarity of the luminescence and ODMR spectra for $\mathrm{KCl}: \mathrm{AgCl}$ (sample 1) and for bulk $\mathrm{AgCl}$ crystal was observed. The principal axes of the STH and the STE in $\mathrm{AgCl}$ microcrystals coincide with the $\langle 100\rangle$ axes of $\mathrm{KCl}$, which proves that the $\mathrm{AgCl}$ microcrystals in $\mathrm{KCl}$ retain the symmetry of the matrix. The misorientation of the $\mathrm{AgCl}$ microcrystals as estimated from the ODMR spectra is below $5^{\circ}$.

The ODMR lines which are marked as STE* in Fig. 10b are attributed to the triplet state of the STE in $\mathrm{AgCl}$ nanocrystals [23, 37-40]. This conclusion was confirmed by the analysis of angular dependences of the ODMR spectra in the (110) 
and (100) planes and by the observation of the forbidden $\Delta M= \pm 2$ transitions typical for an $S=1$ system. The STE* have axial symmetry with the $z$-axis along $\langle 001\rangle$. The nanocrystal size was estimated using atomic force microscopy of a freshly cleaved surface of $\mathrm{KCl}: \mathrm{AgCl}$, which allowed one to measure the size distribution of a nanocrystal [37]. The mean nanocrystals size was found to be about $5-6 \mathrm{~nm}$ in $\mathrm{KCl}: \mathrm{Ag}$ grown with $2 \% \mathrm{AgCl}$. In addition to the STE* ODMR, an axial center with $S=1 / 2$ was observed and tentatively ascribed to $\mathrm{STH}$ in $\mathrm{AgCl}$ nanocrystals [38, 40]. The $g$-factor of SEC in $\mathrm{AgCl}$ nanocrystals was calculated using the $g$-factors of SEC and STH. The obtained parameters for the STH, STE and $\mathrm{SEC}$ in $\mathrm{AgCl}$ micro- and nanocrystals in $\mathrm{KCl}$ crystals are summarized in Table 5 where the data for bulk $\mathrm{AgCl}$ are also presented for comparison. The $95 \mathrm{GHz}$ ODMR in $\mathrm{AgCl}$ nanocrystals embedded in crystalline $\mathrm{KCl}$ was reported in Ref. [41]. Somewhat different results of this study could be connected with a definite difference in the size distribution of the embedded $\mathrm{AgCl}$ crystals.

It is seen from Table 5 that for $\mathrm{AgCl}$ nanocrystals in the $\mathrm{KCl}$ matrix, the fine structure splitting parameter $D$ is much smaller than for bulk $\mathrm{AgCl}$ and the $g$-factor anisotropy $g_{\|}-g_{\perp}$ for both the isolated STH and the STH forming the STE is substantially reduced compared to that for bulk $\mathrm{AgCl}$ crystals. Since the $g$-factor anisotropy is due to the Jahn-Teller effect (JTE) this implies a considerable suppression of the JTE in nanoparticles. As was proposed in Refs. [39, 40], vibronic coupling at the interface brings about weakening or suppression of the JTE in sufficiently small-sized nanocrystals $(L<10 \mathrm{~nm}$ for $\mathrm{AgCl}$ in $\mathrm{KCl})$. This results in a decrease or suppression of the anisotropy in the EPR (ODMR) spectra of the STH; i.e., the difference $g_{\|}-g_{\perp}$ decreases. Inasmuch as the STE is formed through electron capture by the STH, the anisotropy of the g-tensor of the STE should also decrease or be suppressed. It is this decrease that was observed for the anisotropy of ODMR spectra of $\mathrm{AgCl}$ nanocrystals. It should be noted that the suggested interfacial vibronic mechanism of generating inner stress fields in nanocrystals [39, $40]$ is quite general and may be operative for the other nanostructures and thin films.

As was mentioned above, the negative sign of the zero-field splitting parameter $D$ for STE in bulk $\mathrm{AgCl}$ crystals implies that the dominant contribution to the zerofield splitting in the triplet state of the STE is second-order spin-orbit coupling. A strong decrease of the absolute value $D$, which is observed in $\mathrm{AgCl}$ nanocrystals,

Table 5 Parameters of the STH, STE and SEC in bulk $\mathrm{AgCl}$ crystals and $\mathrm{AgCl}$ micro- and nanocrystals embedded in the $\mathrm{KCl}$ crystalline matrix

\begin{tabular}{|c|c|c|c|c|c|c|c|}
\hline \multirow[t]{2}{*}{ Crystal } & \multicolumn{2}{|l|}{ STH } & \multicolumn{3}{|l|}{ STE } & \multirow{2}{*}{$\begin{array}{l}\text { SEC } \\
g\end{array}$} & \multirow[t]{2}{*}{ Refs. } \\
\hline & $g_{\|}$ & $g_{\perp}$ & $g_{\|}$ & $g_{\perp}$ & $D(\mathrm{MHz})$ & & \\
\hline Bulk AgCl & 2.147 & 2.040 & 2.021 & 1.968 & -710 & 1.881 & {$[13,15]$} \\
\hline \multicolumn{8}{|c|}{$\mathrm{AgCl}$ embedded in $\mathrm{KCl}$} \\
\hline Microcrystals & 2.147 & 2.040 & 2.020 & 1.966 & -710 & $1.88-1.90$ & [38-42] \\
\hline \multirow[t]{2}{*}{ Nanocrystals } & & & 1.992 & 1.964 & -335 & & [37] \\
\hline & 2.016 & 1.974 & 1.992 & 1.965 & -335 & $\sim 1.96$ & [38-42] \\
\hline
\end{tabular}


may be due to an increased contribution of the dipole-dipole interactions because of spatial confinement.

\section{2 $\mathrm{AgBr}$ Micro- and Nanocrystals in $\mathrm{KBr}$}

The phenomenon of spatial confinement of the electron-hole recombination in exchange-coupled pairs of recombining SEC and localized holes was revealed in $\mathrm{AgBr}$ nanocrystals embedded in the $\mathrm{KBr}$ matrix by ODMR [23, 42, 43]. It was found that the samples cleaved from different parts of $\mathrm{KBr}$ :Ag crystals grown from the melt (1-2 mol\% $\mathrm{AgBr}$ ) by the Bridgman technique contain self-organized $\mathrm{AgBr}$ microcrystals and/or nanocrystals [42, 43]. Similar to the $\mathrm{AgCl}$ microcrystals in $\mathrm{KCl}$, the $\mathrm{AgBr}$ microcrystals in $\mathrm{KBr}$ retain the properties of bulk $\mathrm{AgBr}$ crystals. Formation of the $\mathrm{AgBr}$ nanocrystals in the $\mathrm{KBr}$ matrix was confirmed by the appearance of a characteristic peak of the exciton emission in the luminescence spectra and its blue shift relative to the exciton emission in bulk $\mathrm{AgBr}[37,42,43]$. $\mathrm{AgBr}$ is an indirect band-gap material and the exciton emission is very weak. The exciton emission intensity increases more than $10^{4}$ times in $\mathrm{AgBr}$ nanocrystals because of modification of the selection rules. A blue shift of the exciton emission appears because of spatial confinement effects. The value of the shift increases with a decrease in the nanocrystal size and can be used for characterization of the size. These effects were observed before for $\mathrm{AgBr}$ nanocrystals dispersed in gelatine, reverse micelles, etc. (see Refs. [44, 45] and references therein). Formation of AgBr microand nanocrystals in glass was reported in Ref. [46] where a single ODMR line was observed for nanocrystals, which corresponds to a strong electron-hole exchange.

Figure 11 presents the PL spectra (Fig. 11a) and the $35 \mathrm{GHz}$ ODMR spectra (Fig. 11b) of $\mathrm{AgBr}$ nanocrystals in $\mathrm{KBr}(1 \% \mathrm{AgBr}$ in the melt) and, for comparison,
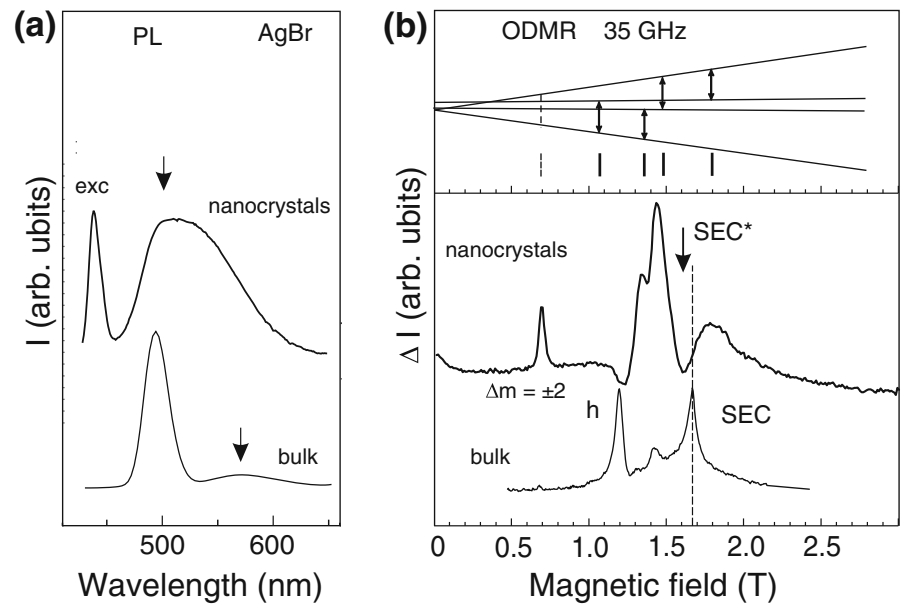

Fig. 11 a PL and b $35 \mathrm{GHz}$ ODMR spectra of $\mathrm{AgBr}$ nanocrystals in $\mathrm{KBr}(1 \% \mathrm{AgBr}$ in the melt) and in the bulk $\mathrm{AgBr}$ crystal. Arrows in a mark the wavelengths at which the ODMR spectra were measured. The energy levels and the $35 \mathrm{GHz}$ EPR transitions for an exchange-coupled pair $\left(J=0.2 \mathrm{~cm}^{-1}\right)$ are shown at the upper part of b. $T=1.8 \mathrm{~K} ; v=35.2 \mathrm{GHz} ; P=300 \mathrm{~mW} ; f_{\mathrm{m}}=80 \mathrm{~Hz}$ 
in the bulk $\mathrm{AgBr}$ crystal, which was used for doping $\mathrm{KBr}$. The energy levels and the $35 \mathrm{GHz}$ transitions for an exchange-coupled pair are shown in the upper part of Fig. 11b. The ODMR spectra were measured at $1.8 \mathrm{~K}$ as microwave-induced variations of the emission intensity at the wavelengths marked by arrows in Fig. 11a. For AgBr nanocrystals, the shape of the observed PL and ODMR spectra is very different from that for nanocrystals. In the PL spectrum of $\mathrm{AgBr}$ nanocrystals a strong emission band of excitons at about $445 \mathrm{~nm}$ is observed, which is shifted relative to the excitons in bulk $\mathrm{AgBr}(461 \mathrm{~nm})$.

In bulk $\mathrm{AgBr}$, the ODMR spectrum is dominated by the lines of the SEC at $g=1.49$ and the localized holes (h) at $g=2.08$. Similar spectra were observed for the $\mathrm{AgBr}$ microcrystals in $\mathrm{KBr}$. The shape of the ODMR spectrum of $\mathrm{AgBr}$ nanocrystals significantly changes with an increase of the microwave chopping frequency and microwave power: the separation of the maxima of the broad signals in the low and high fields increases, while the distance between the central ODMR peaks decreases. Such variations in the shape of ODMR spectra imply that they belong to exchange-coupled recombining electron-hole pairs with a distribution of the interpair separations.

The shape of the ODMR line of shallow donors in bulk semiconductors was analyzed in terms of exchange interactions in the recombining donor-acceptor (D-A) pairs with a random distribution of interpair separations in Ref. [47]. For distant pairs $J=0$, therefore the positions of the ODMR lines correspond to the EPR transitions of the isolated donors and acceptors. Large wings of the ODMR line were explained by a superposition of the ODMR signals from the exchange-coupled D-A pairs with different exchange splittings. The ODMR spectrum of the $\mathrm{AgBr}$ nanocrystals is very different. Since the maximum separation between the recombining centers is limited to the nanocrystal size, there exist no distant pairs in nanocrystals. Therefore, the minima of the ODMR amplitude are expected at the magnetic fields corresponding to $g$-factors of the isolated SEC and hole centers. Such a minimum for SEC in the ODMR spectrum of $\mathrm{AgBr}$ nanocrystals shown in Fig. 11 b corresponds to $g=1.57$, which is different from $g=1.49$ for SEC in bulk $\mathrm{AgBr}$. The $g$-factor shift towards the free-electron value with a decrease of the size of nanoparticles is caused by the confinement of the hydrogen-like $1 s$-type wave function of shallow donors ( $\mathrm{SEC}$ in the case of $\mathrm{AgBr}$ ). It appears when the size of the nanoparticles becomes comparable to the Bohr radius. A similar effect was observed in colloidal $\mathrm{ZnO}$ nanocrystals and was explained by the reduction of the admixture of valence-band states and higher-lying conduction bands into the lowest conduction band due to the increase of the band-gap energy and of the energy of higher-lying conduction bands upon the reduction of the size of the nanocrystals (see Ref. [48] and references therein).

Figure 12a shows a part of the ODMR spectrum of $\mathrm{AgBr}$ nanocrystals recorded by monitoring the total luminescence. The resonance fields $B$ calculated using the spin Hamiltonian Eq. (1) for various exchange interactions $J$ in an exchangecoupled pair of the recombining hole $(g=2.08)$ and SEC $(g=1.57)$ and a relative number $N$ of the recombining pairs as a function of $J$ obtained from the shape of the ODMR spectrum are shown in Fig. 12b. With the assumption that $J$ depends exponentially on the interpair separation and with the Bohr radius of SEC measured 
(a)

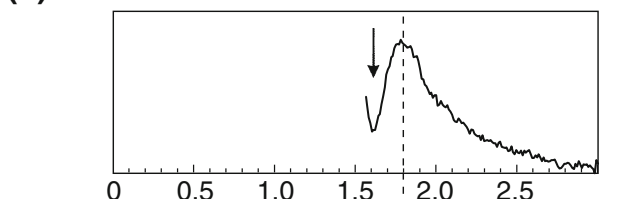

(b)

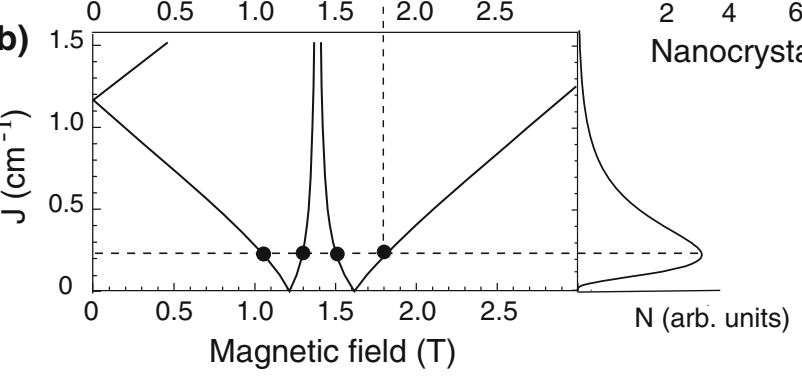

(c)

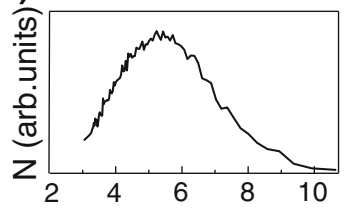

Nanocrystal size $(\mathrm{nm})$

Fig. 12 a Part of the ODMR spectrum recorded by monitoring the total emission of $\mathrm{AgBr}$ nanocrystals in $\mathrm{KBr}$. b Resonance fields $B$ corresponding to the EPR transitions at $35.2 \mathrm{GHz}$ calculated as a function of the exchange splitting $J$ for a pair of the recombining centers with spins $1 / 2$ and $g$ values of 2.08 and 1.57. The right-hand curve shows the relative number $N$ of the recombining pairs as a function of $J$. c Nanocrystal size distribution obtained from the ODMR spectrum

by ENDOR (Sect. 3), the interpair separation can be obtained from the value of $J$. Supposing that the centre of the wave function of SEC is located at the center of the nanocrystal and the holes are localized near the interface, the size distribution of the $\mathrm{AgBr}$ nanocrystals in $\mathrm{KBr}$ was obtained [43]. The size distribution of $\mathrm{AgBr}$ nanocrystals in the sample under study is shown in Fig. 12c. The mean nanocrystal size is estimated to be about $5.5 \mathrm{~nm}$, which is in good agreement with the estimates made on the basis of the blue shift of the exciton emission peak.

\section{Conclusions}

In conclusion, the results of the study of STE, SEC and STH in silver halide crystals by means of high-frequency EPR, ESE, ENDOR and ODMR were discussed.

Application of high-frequency $(95 \mathrm{GHz})$ and multiquantum $(35 \mathrm{GHz})$ ODMR allowed to reveal the ordering of the singlet and triplet levels of the STE in $\mathrm{AgCl}$ and to measure their splitting with very high accuracy: $J=5.370 \pm 0.002 \mathrm{~cm}^{-1}$. The dynamical properties of the excited singlet and triplet states of the STE were studied by ESE technique in combination with pulsed laser excitation. The populating, depopulating and spin-lattice relaxation processes have been studied.

The presented ENDOR study demonstrates that the lowest triplet state of the STE in $\mathrm{AgCl}$ consists of a very diffuse electron attracted by a strongly localized STH. It was found that the spatial distribution of the STE can roughly be divided into three regions. At distances larger than about $18 \AA$, the wave function of the STE is completely determined by the shallow electron, which occupies a hydrogen-like $1 s$ orbital with a Bohr radius of $15.1 \pm 0.6 \AA$. At distances between 18 and $9 \AA$, the electron is still dominant but its density distribution deviates from the spherical 
symmetry and reflects the $D_{4 h}$ symmetry of the central STH. At distances shorter than about $9 \AA$, both the central hole and the shallow electron have a contribution to the wave function of the STE.

The ENDOR studies allowed the direct determination of the wave function of the intrinsic SEC and STH in silver chloride. A model of SECs is suggested in which the electron is shallowly trapped in the Coulombic field of two adjacent $\mathrm{Ag}^{+}$ions, symmetrically placed on a single cationic site. The ENDOR studies of STH provide direct information about the spatial distribution of the unpaired electron at the silver and chlorine sites and of the electrical field gradient distribution at the chlorine sites of the STH. From a comparison of the ENDOR results for STE and STH, it is concluded that the central hole of the STE is virtually identical to an isolated STH center.

Self-organized micron-size and nanometer-size $\mathrm{AgCl}$ and $\mathrm{AgBr}$ crystals (microcrystals and nanocrystals) embedded in the $\mathrm{KCl}$ and $\mathrm{KBr}$ crystalline matrices and retaining the orientation of the host lattice were studied by ODMR. For $\mathrm{AgCl}$ nanocrystals in $\mathrm{KCl}$, the anisotropy of the $g$-factor for both the isolated STH and the STH forming the STE is found to be substantially reduced compared to that in bulk $\mathrm{AgCl}$ crystals. This implies a considerable suppression of the Jahn-Teller effect in nanoparticles and can be explained by a model in which an additional deformation field appearing because of the strong vibronic interaction at the interface is taken into account. The spatial confinement effects were found in $\mathrm{AgBr}$ nanocrystals embedded in the $\mathrm{KBr}$ crystalline matrix. They are manifested by the $g$-factor shift for SECs and the maximum distance between the recombining SEC and hole centers being restricted to the nanocrystal size.

Acknowledgments We are indebted to M.T. Bennebroek and A.v. Duijn-Arnold for collaboration. This work has been supported by the Ministry of Education and Science, Russia, under the contracts nr. 02.740.11.0108 and 14.740.11.0048; the Programs of the Russian Academy of Sciences: "SpinDependent Effects in Solids and Spintronics", "Support of Innovations and Elaborations", "Basic Researches of Nanotechnologies and Nanomaterials" and by the Russian Foundation for Basic Research under grants nr. 09-02-01409 and 09-02-00730.

Open Access This article is distributed under the terms of the Creative Commons Attribution Noncommercial License which permits any noncommercial use, distribution, and reproduction in any medium, provided the original author(s) and source are credited.

\section{References}

1. L.D. Landau, Phys. Z. Sowjetunion 3, 664 (1933)

2. M.N. Kabler, in Point Defects in Solids, ed. by J.H. Crawford Jr., L.M. Slifkin (Plenum Press, New York, 1972)

3. K.S. Song, R.T. Williams, Self-Trapped Excitons, chapt. 9 (Springer, Heidelberg, 1993)

4. W. Känzig, Phys. Rev. 99, 1890 (1955)

5. T.G. Castner, W. Känzig, J. Phys. Chem. Solids 3, 178 (1957)

6. R. Gazzinelli, R.L. Mieher, Phys. Rev. 175, 395 (1968)

7. M. Höhne, M. Stasiw, Phys. Status Solidi 25, k55 (1968)

8. M. Höhne, M. Stasiw, Phys. Status Solidi 28, 247 (1968)

9. M. Fukui, Y. Hayashi, H. Yoshioka, J. Phys. Soc. Jpn. 34, 1226 (1973) 
10. H. Kanzaki, Photogr. Sci. Eng. 24, 219 (1980)

11. W. Hayes, I.B. Owen, J. Phys. C 9, L69 (1976)

12. K. Murayama, K. Morigaki, S. Sakuragi, H. Kanzaki, J. Phys. Soc. Jpn. 41, 1617 (1976)

13. W. Hayes, I.B. Owen, P.J. Walker, J. Phys. C 10, 1751 (1977)

14. W. Hayes, I.B. Owen, J. Phys. C 11, L607 (1978)

15. A.P. Marchetti, D.S. Tinti, Phys. Rev. B 24, 7361 (1981)

16. H. Yoshioka, N. Sugimoto, M. Yamaga, J. Phys. Soc. Jpn. 54, 3990 (1985)

17. N. Sugimoto, H. Yoshioka, M. Yamaga, J. Phys. Soc. Jpn. 54, 4331 (1985)

18. M. Yamaga, N. Sugimoto, H. Yoshioka, J. Phys. Soc. Jpn. 54, 4340 (1985)

19. J.P. Spoonhower, F.J. Ahlers, R.S. Eachus, W.G. McDugle, J. Phys. C 2, 3021 (1990)

20. M.C.J.M. Donckers, O.G. Poluektov, J. Schmidt, P.G. Baranov, Phys. Rev. B 45, 13061 (1992)

21. O.G. Poluektov, M.C.J.M. Donckers, P.G. Baranov, J. Schmidt, Phys. Rev. B 47, 10226 (1993)

22. N.G. Romanov, P.G. Baranov, Semicond. Sci. Technol. 9, 1080 (1994)

23. P.G. Baranov, N.G. Romanov, Appl. Magn. Reson. 21, 165 (2001)

24. M.T. Bennebroek, O.G. Poluektov, A.J. Zakrzewsky, P.G. Baranov, J. Schmidt, Phys. Rev. Lett. 74, 442 (1995)

25. M.T. Bennebroek, A. Arnold, O.G. Poluektov, P.G. Baranov, J. Schmidt, Phys. Rev. B 53, 15607 (1996)

26. M.T. Bennebroek, A. Arnold, O.G. Poluektov, P.G. Baranov, J. Schmidt, Phys. Rev. B 54, 11276 (1996)

27. M.T. Bennebroek, A.v. Duijn-Arnold, J. Schmidt, O.G. Poluektov, P.G. Baranov, Phys. Rev. B 66, 054305 (2002)

28. A.M. Stoneham, Theory of Defects in Solids (Clarendon, Oxford, 1985)

29. R.C. Baetzold, R.S. Eachus, J. Phys. C 7, 3991 (1995)

30. B.S. Gourary, F.J. Adrian, Phys. Rev. 105, 1180 (1957)

31. R.A. Zhitnikov, P.G. Baranov, N.I. Melnikov, Phys. Status Solidi (B) 59, K111 (1973)

32. R.S. Eachus, R.E. Graves, M.T. Olm, Phys. Status Solidi (B) 152, 583 (1989)

33. S. Sakuragi, H. Kanzaki, Phys. Rev. Lett. 38, 1302 (1977)

34. V.G. Grachev, Sov. Phys. JETP 65, 1029 (1987)

35. M. Moreno, J. Phys. Chem. Solids 51, 835 (1990)

36. R. Valiente, J.A. Aramburu, M.T. Barriuso, M. Moreno, J. Phys. C 6, 4515 (1994)

37. H. Vogelsang, O. Husberg, U. Köhler, W. von der Osten, A.P. Marchetti, Phys. Rev. B 61, 1847 (2000)

38. P.G. Baranov, N.G. Romanov, V.A. Khramtsov, A.G. Badalyan, R.A. Babunts, Phys. Solid State 42(12), 2231-2235 (2000) (Translated from Fizika Tverdogo Tela, 42(12), 2166-2170 (2000))

39. P.G. Baranov, V.S. Vikhnin, N.G. Romanov, V.A. Khramtsov, JETP Lett. 72, 329 (2000)

40. P.G. Baranov, V.S. Vikhnin, N.G. Romanov, V.A. Khramtsov, J. Phys. Condens. Matter 13, 2651 (2001)

41. G. Janssen, A. Bouwen, E. Goovaerts, Phys. Rev. B 71, 035415 (2005)

42. P.G. Baranov, N.G. Romanov, V.L. Preobrazhenskii, V.A. Khramtsov, JETP Lett. 76, 465 (2002)

43. P.G. Baranov, N.G. Romanov, V.A. Khramtsov, V.S. Vikhnin, Nanotechnology 12, 540 (2002)

44. A.P. Marchetti, K.P. Johanson, G.L. McLendon, Phys. Rev. B 47, 4268 (1993)

45. P.J. Rodney, A.P. Marchetti, P.M. Fauchet, Phys. Rev. B 62, 4215 (2000)

46. A.P. Marchetti, P.J. Rodney, W. von der Osten, Phys. Rev. B 64, 132201 (2001)

47. J.M. Trombetta, T.A. Kennedy, Phys. Rev. B 48, 17031 (1993)

48. D.M. Hofmann, H. Zhou, D.R. Pfisterer, H. Alves, B.K. Meyer, P. Baranov, N. Romanov, C. de Mello Donega, A. Meijering, S. Orlinskii, H. Blok, J. Schmidt, Phys. Status Solidi C 1, 908 (2004) 